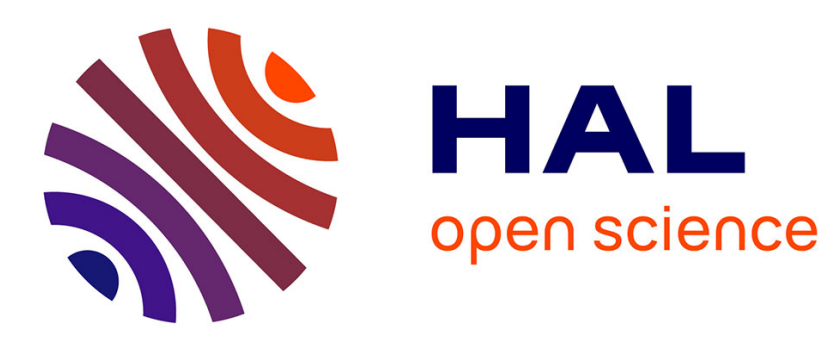

\title{
ECaD: Energy-efficient routing in flying ad hoc networks
}

Omar Sami Oubbati, Mohammad Mozaffari, Noureddine Chaib, Pascal

Lorenz, Mohammed Atiquzzaman, Abbas Jamalipour

\section{To cite this version:}

Omar Sami Oubbati, Mohammad Mozaffari, Noureddine Chaib, Pascal Lorenz, Mohammed Atiquzzaman, et al.. ECaD: Energy-efficient routing in flying ad hoc networks. 2019. hal-02320676

\section{HAL Id: hal-02320676 \\ https://hal.science/hal-02320676}

Submitted on 19 Oct 2019

HAL is a multi-disciplinary open access archive for the deposit and dissemination of scientific research documents, whether they are published or not. The documents may come from teaching and research institutions in France or abroad, or from public or private research centers.
L'archive ouverte pluridisciplinaire HAL, est destinée au dépôt et à la diffusion de documents scientifiques de niveau recherche, publiés ou non, émanant des établissements d'enseignement et de recherche français ou étrangers, des laboratoires publics ou privés. 


\title{
ECaD: Energy-Efficient Routing in Flying Ad hoc Networks
}

\author{
Omar Sami Oubbati, Member, IEEE, Mohammad Mozaffari, Member, IEEE, \\ Noureddine Chaib, Senior Member, IEEE, Pascal Lorenz, Senior Member, IEEE, \\ Mohammed Atiquzzaman, Senior Member, IEEE, and Abbas Jamalipour, Fellow, IEEE
}

\begin{abstract}
Much progress can be expected in the domain of Unmanned Aerial Vehicle (UAV) communication by the next decade. The cooperation between multiple UAVs in the air exchanging data among themselves can naturally form a Flying Ad hoc Network (FANET). Such networks can be the key support to accomplish several kinds of missions while providing the required assistance to terrestrial networks. However, they are confronted with many challenges and difficulties, which are due to the high mobility of UAVs, the frequent packet losses, and the weak links between UAVs, all affecting the reliability of the data delivery. Furthermore, the unbalanced energy consumption may result in earlier UAV failure, and consequently, accelerate the decrease of the network lifetime, thus disrupting the overall network. This paper supports the use of the movement information and the residual energy level of each UAV to guarantee a high level of communication stability while predicting a sudden link breakage prior to its occurrence. A robust route discovery process is used to explore routing paths where the balanced energy consumption, the link breakage prediction, and the connectivity degree of the discovered paths are all considered. The performance of the scheme is evaluated through a series of simulations. The outcomes demonstrate the benefits of the proposed scheme in terms of increasing the lifetime of the network, minimizing the number of path failures, and decreasing the packet losses.
\end{abstract}

Index Terms-Routing, Energy efficiency, UAV, Connectivity, Simulation, Flooding process.

\section{INTRODUCTION}

Equipped with wireless communication modules and appropriate sensors, Unmanned Aerial Vehicles (UAVs) can be used as a single connected group and deployed in many civilian and military applications [1]. UAVs are characterized by their high mobility and their adjustable altitudes making them the appropriate candidates to be elected as wireless relays to improve the connectivity and to extend the coverage of a specific area [2], [3]. As a result, the cooperation in an ad hoc fashion between several UAVs to form a Flying Ad hoc Network (FANET) is becoming widely applicable, since most of the UAV applications require more flexibility and

O. S. Oubbati \& N. Chaib are with the Computer Science and mathematics Laboratory, University of Laghouat, BP 37G, Ghardaïa Road, Laghouat 03000, Algeria. E-mail: \{s.oubbati, n.chaib\}@lagh-univ.dz.

M. Mozaffari is with Electrical and Computer Engineering Department, Virginia Tech, Blacksburg, VA, USA. E-mail: mmozaff@ vt.edu.

P. Lorenz is with is with University of Haute Alsace, France.

E-mail: pascal.lorenz@uha.fr.

M. Atiquzzaman is with the University of Oklahoma, Norman, OK USA. E-mail: atiq@ou.edu.

A. Jamalipour is with School of Electrical and Information Engineering, University of Sydney, Camperdown NSW 2006, Australia.

E-mail: a.jamalipour@ieee.org. versatility in order to be easily deployed [4], [5]. Nevertheless, FANETs face numerous challenges, such as the frequent topology changes in a 3D space due to the high-speed ranges of UAVs resulting in unstable communications between them (i.e., communicating UAVs stay in range for a short period of time) [6]. Another challenging issue is that the lifetime of FANETs is strongly related to the energy constraints imposed by the weight, size, and power consumption of UAVs [7]. Moreover, the energy capacity of UAVs is highly restricted by their on-board batteries that are unable to power UAVs all the time.

Coming soon, the emergence of the fifth generation (5G) wireless systems can be a key support to FANET real-time applications in order to ensure stable and uninterrupted communications [8]. However, some other kinds of applications have a basic need of coordination between UAVs to be carried out efficiently, and in most cases, in a timely manner (e.g., rescue and search missions in disaster areas). Moreover, UAVs can be further exploited in the e-commerce industry [9]. Two critical problems arise: (i) weak connection links between UAVs due to their high mobility, where they can be subjected to packet losses and (ii) the limited energy capacity of UAVs, where the communication links would be frequently interrupted due to the insufficient transmission power. These problems are not simple to be solved due to the unique characteristics of FANETs, such as the dynamic altitude of UAVs, the adopted mobility models, and the network fragmentations [10]. No existing routing FANET solution has resolved these two problems together. For instance, the work in [11] organizes the network into clusters in order to reduce the energy consumption. The next hop cluster-heads (CHs) are selected based on many criteria, such as their energy levels at a given time and the distance separating them from the destination. Nevertheless, this technique does not consider the connectivity degree of the links and, in the case of disconnections, no recovery strategy is adopted. Moreover, as many communications transit via the cluster heads, they tend to be quickly lacking energy and may stop functioning. This disrupts the overall network and causes routing failures. In [12], a trade-off between the optimal number of UAVs to communicate with the destination (base station) and the energy-efficient communication links among the UAVs, in order to achieve optimal energy management. However, this technique does not use any connectivity measurement and it assumes that the UAV can reach any UAV using one-hop communication, which is not conceivable and realistic since 
all UAVs have a limited transmission range and they can be far from each other.

Several other energy-efficient proposals adopted in other kinds of networks, such as Wireless Sensor Networks (WSNs) have been proposed in the literature. For instance, the work in [13] increases the wireless network performance by combining an energy-aware simultaneous wireless information and power transfer (SWIPT) routing algorithm with a multi-hop wireless strategy. The authors in [14] proposed an energy-efficient coverage control technique using Practical swarm optimization (PSO) for WSNs. This approach divides an area that comprises several sensors into multiple grids and adjusting their sensing radius according to their energy consumption. Similarly, an energy efficient routing scheme based on clustering is proposed in [15]. Indeed, each $\mathrm{CH}$ is elected based on two metrics, such as the residual energy and the distance that separates it from the source. The communications between the source nodes and their $\mathrm{CHs}$ are carried out using a single or multi-hop communication and the $\mathrm{CHs}$ that the closest ones to the mobile sink are selected to communicate with it. In another context, the work in [16] established the networking of wearable devices (i.e., Micro WSN) using the concept of minimal connected dominating sets (MCDSs) to organize nodes, establish a virtual backbone, transmit data to rescue teams, and reduce the energy consumption of the network. In [17], a combination of the problem of source, routing selection, and channel assignment in multi-source transmission in the interests of improving the throughput.

To overcome the different aforementioned issues and to be more inspired by the discussed proposals, a reliable routing strategy has to be defined while considering the different challenges of FANETs. The key ideas behind this routing strategy is to exploit UAVs in the network to efficiently anticipate path failures before their occurrence and select alternative next hops. Moreover, the strategy should have the ability to establish routing paths comprising UAVs with higher residual levels of energy while excluding UAVs with a low energy capacity. This allows to avoid that some UAVs will be unfairly burdened and to spare them from any participation in the data delivery, while excluding some exceptional cases. In response to these goals, a robust reactive routing protocol is proposed, namely ECaD (Energy-efficient Connectivity-aware Data Delivery). The novelties of ECAD's scheme can be summarized as follows:

- To strengthen the established routing paths, a discovery process inspired from AODV [18] is adopted, which is enhanced to exploit control messages to have a global vision about the connectedness degree of the links and the energy levels of UAVs.

- To make the routing fault tolerant, an efficient maintenance process is used, which both avoids the reinitialization of the discovery process when there are unavoidable disconnections and provides alternative solutions to reach the destinations.

- To limit the overhead, a set of measures is taken into account, such as the use of static size routing packets to avoid recording the full routing paths and the necessity to avoid introducing both additional delays and a large amount of network traffic by minimizing the frequency of flooding.

To better understand the important processes of ECaD and its functionality, several contributions have been made in this paper as follows:

- We conducted a series of descriptions of the most relevant routing protocols in the literature along with their drawbacks. Also, we carry out a comparative study to examine the deficiencies between the studied protocols and our proposed scheme.

- We provided a detailed depiction of the different processes modeling the proposed scheme and how they are combined to provide an efficient and reliable routing path dealing with the energy restriction of UAVs.

- We validated the proposed protocol by studying its costs in terms of complexity after proving its loop freedom property.

- We performed a series of simulations to study the realistic effects of FANET environments on our proposed protocol. The obtained results show the efficiency of ECaD to deal with the unregulated energy consumption of UAVs and the frequent disconnections.

The rest of the paper is structured as follows. Section II reviews a series of related routing protocols which are relevant to our work. Section III presents the technical details of our work. Section IV provides a theoretical analysis of our proposed routing protocol. Section $\mathrm{V}$ verifies the effectiveness of our work based on simulation results. Finally, Section VI draws conclusions on this work and some future perspectives.

\section{RELATED WORK}

Research on routing protocols targeting FANET are still at its infancy, especially in the area of energy-efficient strategies. In this section, a review of the existing routing solutions dedicated for FANETs will be limited only to those adopting the route discovery, the greedy forwarding, the position prediction, and the energy efficiency. These categories are considered as the most relevant to shape our own routing protocol.

The route discovery is adopted only when a UAV wants to engage a communication and the position of the destination is supposed to be unknown. In [19], the route discovery tries to find the shortest path to the destination (i.e., minimum of hops). The novelty is that the destination's position is included in the route reply (RREP) packet and it is shared with all intermediate nodes. When there is a disconnection, the greedy forwarding technique is used by UAVs until the destination. As a drawback, the links composing the selected path can be quickly broken since any connectivity factor is taken into account. This results in many route discoveries, and thus consuming more resources and energy. To address these issues, the work presented in [20] performs path discovery on-demand taking into account the connectivity factor among UAVs. Indeed, the sequence of UAVs close to each other is desirable to build a robust routing path. However, this protocol cannot deal with sudden link breakages occurred on multiple links constituting the path, since it cannot find several alternative solutions at once. 
When a network is highly dense and the destination's position is supposed to be known, the greedy forwarding is ideally suited. Nevertheless, the connectedness of FANET is not permanent since UAVs are highly mobile, which makes the greedy forwarding unreliable when it is misused. In [21], the mobility of UAVs follows the Gaussian Markov model which can help to easily predict the positions of neighboring UAVs, and thus the optimal forwarder towards the destination. In the case of multiple link failures, this technique cannot continue to operate normally causing severe packet losses. In [22], a prediction method based on the Gaussian distribution is used to predict the geographic position of a UAV at a given time $t_{n}$. This allows analyzing the persistent connection of the neighboring UAVs. In the case when any forwarder is found, the distance between each two hops neighbor and the destination is calculated in order to find another forwarder to the target destination. This technique can fail when the selected forwarder UAVs are in their low battery levels.

There is a necessity to predict how long wireless links would last between UAVs, and especially when UAVs are deployed in a highly dynamic 3D scenario. The authors in [23] developed a new variant of OLSR protocol to predict the link quality between UAVs. This technique favors UAVs that remain stable and close to each other during the routing phase. However, at each change of the topology, updates are broadcasted all over the network causing heavy traffic, and thus more energy consumption. To handle these issues, the work presented in [24] aims to compute multiple stable link-disjoint paths (i.e., different paths share no common links). In addition, a link stability metric is calculated by the source UAV to select the most stable path among the multiple link-disjoint cached paths. In the case of disconnections, the most stable cached path is selected for the data transmission. When the network is highly dense of UAVs, it can cause an extensive use of memory (e.g., storage of huge routing tables), thus consuming an important energy.

Unbalanced energy consumption among UAVs is considered as a serious problem. In [25], the network is organized into clusters, where a $\mathrm{CH}$ is elected based on the energy level, the relative velocity, and the degree of connectivity with its members. Member nodes use intra-cluster communications to communicate directly with each other. Otherwise, all communications transit through the $\mathrm{CH}$ since it has a sufficient residual energy to communicate with other $\mathrm{CHs}$ located a little far. Nevertheless, as successive communications transit through the $\mathrm{CH}$, its residual energy tends to be minimized, and it will run out of energy sooner than other UAVs making this strategy fail. To overcome this problem, the work in [26] divides the network into clusters. For the uniform energy consumption, $\mathrm{CHs}$ are re-elected in subsequent rounds. However, this election is based on a probabilistic method using random number generation which is not suitable in the case when a cluster member is already in its critical level of energy. Another strategy is adopted in [11], where the locations of UAVs and their remaining energy levels are considered. The positions of undetermined UAVs are calculated based on the received signal strength indication (RSSI) between them. The energy consumption is improved by organizing the network into clusters. Based on the localization algorithm, the $\mathrm{CHs}$ are elected and can be selected as next hops based on several parameters. Nevertheless, a large amount of overhead is observed during both the formation of the clusters and the election of their CHs. The work in [27] tries to minimize the overhead by keeping the clustering formation along with a higher energy level. The transmission power (i.e., the communication range) is considered to be dynamic according to the distance separating the communicating UAVs. The clusters are formed based on the K-means density (i.e., the degree of the neighborhood) to select the adequate $\mathrm{CH}$. As an inconvenience, this kind of routing protocols works well under a path planned mobility model, which is not the case in most of FANET applications. Other kinds of schemes are proposed to overcome the energy constraints of UAVs and in particular mobile nodes. For instance, the authors in [28] proposed an interesting charging/discharging strategy of electrical vehicles. In fact, this strategy takes into account the residual energy, the user charge habit, and the efficiency of the charging stations, with the aim to enhance the charging demand balance among different charging stations. Another kind of scheme is proposed in [29] where an efficient data gathering scheme based on neural network is applied to conduct the data fusion with the aim to improve the energy efficiency and the network throughput.

None of the prior studies have efficiently addressed the problem of spontaneous disconnections caused by many factors, such as the high mobility, a UAV failure due to its limited energy capacity, or both. Moreover, several realistic characteristics have been neglected during their evaluations. Table I provides a summary of features comparison among the previously described routing protocols with those considered by our routing protocol.

\section{SYSTEM MODEL}

In this work, a team of $n$ UAVs is fairly deployed in a 3D, and initially, the battery of each UAV is considered to be in full level of energy tending to diminish over the time (c.f.,

TABLE I: Features comparison of the related routing protocols.

\begin{tabular}{|c|c|c|c|c|c|c|c|c|c|c|c|}
\hline \multirow{3}{*}{ Features } & \multicolumn{2}{|c|}{ Discovery-based } & \multicolumn{2}{|c|}{ Greedy-based } & \multicolumn{2}{|c|}{ Prediction-based } & \multicolumn{4}{|c|}{ Energy efficient } & \multirow{3}{*}{ Our protocol } \\
\hline & RGR & UVAR & GPMOR & MPGR & P-OLSR & LEPR & CBLADSR & LEACH & IMRL & EALC & \\
\hline & Ref. [19] & Ref. [20] & Ref. [21] & Ref. [22] & Ref. [23] & Ref. [24] & Ref. [25] & Ref. [26] & {$[11]$} & {$[27]$} & \\
\hline Link stability & $\times$ & $\sqrt{ }$ & $\sqrt{ }$ & $\sqrt{ }$ & $\sqrt{ }$ & $\sqrt{ }$ & $\times$ & $\sqrt{ }$ & $\times$ & $\times$ & $\sqrt{ }$ \\
\hline Predictive & $\times$ & $x$ & $\sqrt{ }$ & $\sqrt{ }$ & $x$ & $x$ & $x$ & $x$ & $x$ & $x$ & $\sqrt{ }$ \\
\hline Maintenance & $\sqrt{ }$ & $\sqrt{ }$ & $x$ & $\sqrt{ }$ & $x$ & $\sqrt{ }$ & $\times$ & $x$ & $x$ & $\sqrt{ }$ & $\sqrt{ }$ \\
\hline Discovery & $\sqrt{ }$ & $\sqrt{ }$ & $x$ & $x$ & $x$ & $\sqrt{ }$ & $\times$ & $x$ & $x$ & $x$ & $\sqrt{ }$ \\
\hline Energy efficiency & $x$ & $\times$ & $\times$ & $x$ & $\times$ & $x$ & $\sqrt{ }$ & $\sqrt{ }$ & $\sqrt{ }$ & $\sqrt{ }$ & $\sqrt{ }$ \\
\hline Network & FANET & FANET/VANET & FANET & FANET & FANET & FANET & FANET & WSN & FANET & FANET & FANET \\
\hline
\end{tabular}


Figure 1). UAVs aim to cooperatively achieve a given mission requiring a unicast exchange of crucial messages between each other. To do so, we suppose that each UAV is aware of its own geographical position ( $\mathrm{x}, \mathrm{y}, \mathrm{z}$ ) using GPS and it periodically updates both its routing table and table of neighbors. The links between two UAVs are considered to be bidirectional and operates in the $5 \mathrm{GHz}$ wireless band [30]. IEEE 802.11a wireless interfaces are adopted at the MAC layer of each UAV, since they are considered as an efficient support to highly dynamic topologies and provide a wide coverage of wireless communications [31], [32].

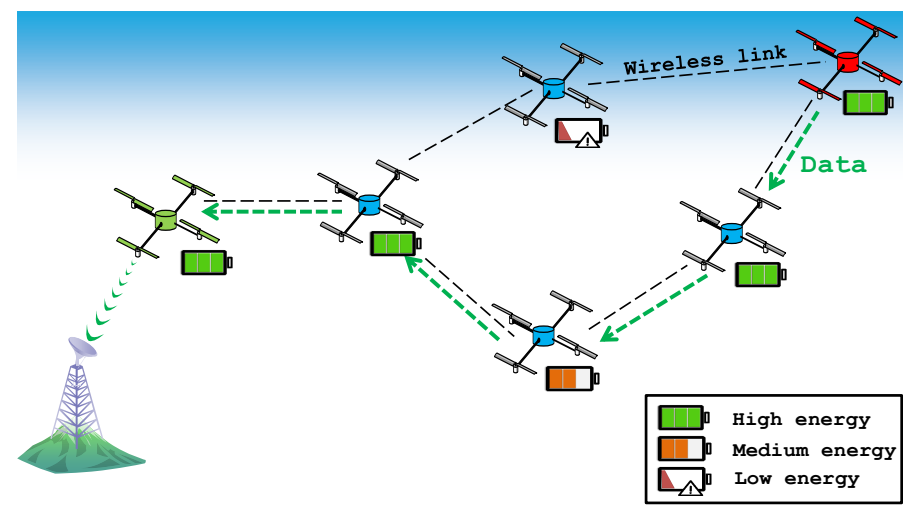

Fig. 1: System model.

Since our system only considers the air-to-air (A2A) channel model, the path loss is dominant with less fading and can be defined by the free-space propagation model. According to [33], the path loss for the air-to-air channel (PLAA) can be expressed as follows:

$$
\operatorname{PLAA}(x)=\beta 10 \log _{10} x+\alpha .
$$

where $\beta$ is the path loss exponent, $x=$ $\sqrt{\left(x_{i}-x_{j}\right)^{2}+\left(y_{i}-y_{j}\right)^{2}+\left(z_{i}-z_{j}\right)^{2}}$ is the distance between the communicating $\mathrm{UAVs} u_{i}$ and $u_{j}, \alpha$ represents the the path loss at the reference point. According to the free-space propagation model, $\beta=2$ and $\alpha=10 \log _{10}\left(\frac{4 \pi w}{l}\right)$; where $w$ is the carrier frequency and $l=3 \times 10^{8} \mathrm{~m} / \mathrm{s}$ is the light speed.

The FANET wireless communication hardwares are powered by the energy resources of UAVs, such as embedded batteries. Nevertheless, due to their weight constraints (or payload), their frequent wireless communications, their mobility, and their small battery sizes, the UAVs have a quite restricted energy capacity [34]. Consequently, these factors can be combined to calculate locally the residual energy of each UAV, but this calculation is out of the scope of this work. It is worthy to note that a threshold $(\tau)$ is set to a certain value to exclude any transmission when the residual energy of UAV $u_{i}\left(R_{u_{i}}\right)$ is below $\tau\left(R_{u_{i}}<\tau\right)$, except in certain cases where $\tau$ can be modified by $u_{i}$ according to the priority of the data packets to send. This priority varies for different kinds of applications. Two fields are added into the Hello packet format, which contain the residual energy and the movement information of each UAV, in order to be aware of the remaining energy level of all neighboring UAVs and to predict any disconnection between them, respectively.
For the sake of clarity, Table II summarizes the major notations used in the rest of the paper.

TABLE II: Summary of notations.

\begin{tabular}{|c|c|}
\hline Notation & Definition \\
\hline $\bar{U}$ & A set of UAVs. \\
\hline$u_{i}$ & A UAV with an identifier $i$. \\
\hline$N\left(u_{i}\right)$ & Direct neighbors of $u_{i}$ \\
\hline & A minimum threshold to make a data transmission. \\
\hline$P_{i}\left(u_{i}, u_{j}\right)$ & Path $i$ from $u_{i}$ to $u_{j}$. \\
\hline$u_{i} u_{j}$ & $\begin{array}{l}\text { Direct link from } u_{i} \text { to } u_{j} \text {, i.e., } u_{i} \text { and } u_{j} \text { are } \\
\text { neighbors. }\end{array}$ \\
\hline$C E$ & Connectivity duration time of a given link. \\
\hline$C E_{\overline{u_{i} u_{j}}}$ & Connectivity duration time of $\overline{u_{i} u_{j}}$. \\
\hline$C E_{P_{i}\left(u_{i}, u_{j}\right)}$ & $\min _{\overline{u_{i+1} u_{i+2}} \in P_{i}\left(u_{i}, u_{j}\right)} C E$ \\
\hline$R$ & Residual energy level of a given UAV. \\
\hline$R_{u_{i}}\left(u_{i}, u_{j}\right)$ & Residual energy level of $u_{i}$ \\
\hline$R_{e r c}$ & Ratio of energy remaining capacity. \\
\hline Delay $\overline{\overline{u_{i} u_{j}}}$ & Required time for a packet to transit $\overline{u_{i} u_{j}}$. \\
\hline $\operatorname{Delay}_{P_{i}}\left(u_{i}, u_{j}\right)$ & Required time for a packet to transit $P_{i}\left(u_{i}, u_{j}\right)$. \\
\hline Delay $_{P}$ & $\begin{array}{l}\text { Required time to transit the path between two com- } \\
\text { municating nodes. }\end{array}$ \\
\hline$C E_{P}$ & $\begin{array}{l}\text { Minimum connectivity expiration time of the path } \\
\text { between two communicating nodes. }\end{array}$ \\
\hline$R_{P}$ & $\begin{array}{l}\text { Lowest residual energy of all succession of UAVs } \\
\text { constituting the path between two communicating } \\
\text { nodes. }\end{array}$ \\
\hline$R D_{i}$ & Routing decision at the level $i$. \\
\hline HOPS & Number of hops. \\
\hline$S_{\overline{u_{i} u_{j}}}$ & Score of the path until $u_{i}$ via the previous hop $u_{j}$. \\
\hline RREQ & Route REQuest. \\
\hline RREP & Route REPly. \\
\hline RERR & Route ERRor. \\
\hline
\end{tabular}

\section{A. ECaD overview}

We propose ECaD (Energy-efficient Connectivity-aware Data Delivery) which is a novel routing protocol based on an energy conserving technique and connectivity measurements. ECaD establishes an on-demand robust routing path between the communicating UAVs while taking into account the different constraints related to both the unstable mobility and the near-optimal exploitation of the energy resources of UAVs. The originality of ECaD is that it builds multiple paths towards the destination while considering at each hop both the robustness of links and the energy of UAVs. As shown in Figure 2, ECaD mainly relies on three processes in order to build, use, and maintain a routing path.

To achieve building, the requesting UAV initiates a discovery process by flooding a route request (RREQ) packet over the network to explore robust and energy-rich paths, while considering each UAV willingness to take part in this process on behalf of other UAVs. Each neighboring UAV $u_{i}$ decides whether to accept broadcasting the intercepted RREQ or not depending on its residual energy $R_{u_{i}}$ and the defined $\tau$. For instance, when $R_{u_{i}}$ is higher than $\tau$, the RREQ is broadcasted, otherwise, the packet is dropped. The flooding results in a gradual construction of multiple routing paths along with a progressive calculation of important parameters, such as the connectivity expiration, the residual energy, and the delay of delivering of each discovered path. Each path is illustrated by a succession of UAVs, which is not stored neither in the control packets (RREQs and RREPs) nor locally in a 


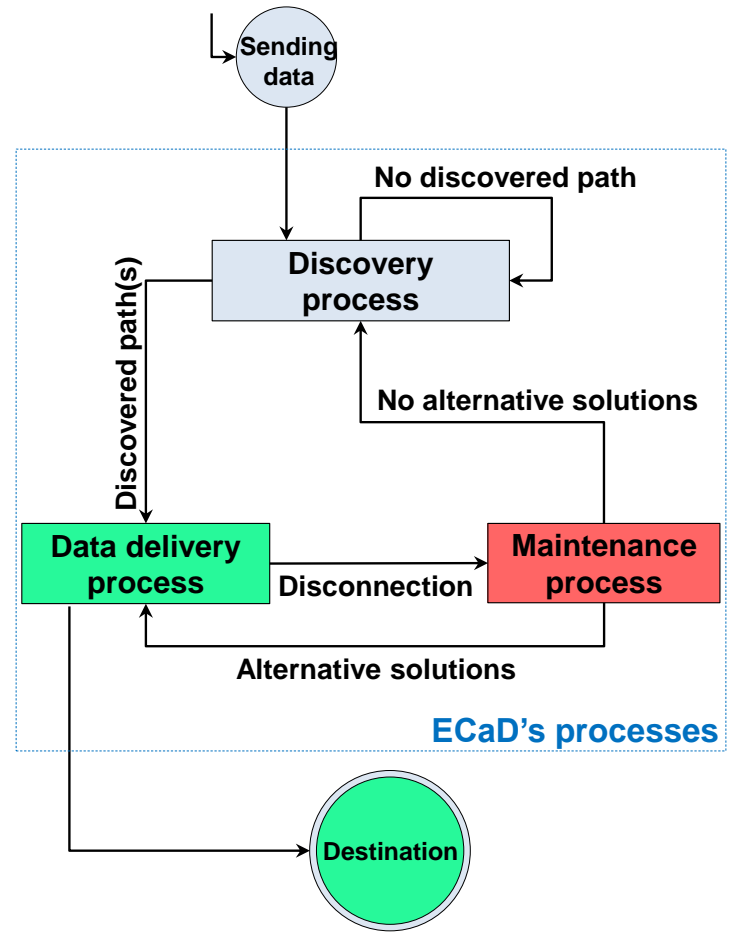

Fig. 2: Processes of ECaD.

limited memory. Consequently, it should be stressed that ECaD uses an enhanced mechanism based on routing tables that are updated and maintained locally at each intermediate UAV.

The destination gets RREQs only when the transited intermediate UAVs constituting the routing paths have a good battery power or at least have a residual level of energy above $\tau$. Therefore, when getting all possible RREQs, a selection is carried out to pick out the most connected routing path with a reduced delay and follows an energy optimization strategy. Once a path is selected, an RREP packet is generated and sent back to the source through this path. The exploitation of the latter is the responsibility of the data delivery process where data packets are transited along the selected succession of UAVs. To be tolerant to possible disconnections, the maintenance process is initiated by the current UAV (i.e., the UAV that detects the failure) to find alternative solutions (i.e., another appropriate next hop) in order to be able to normally continue delivering data packets to their target destinations.

To deeply understand the ECaD's processes, we first need to describe the format of different packets used by ECaD. Then, we provide the calculation method of the connectivity expiration of a given path. After that, we illustrate the energy consumption management among UAVs based on different scenarios. Finally, a concrete example is provided in order to demonstrate the application of the ECaD's functioning.

1) Packet format: The RREQ packet format consists of several fields illustrated in Figure 3. The Communication ID field identifies the discovery process to which the RREQ packet belongs. The Delay $y_{P}$ is defined as the required time to transit the full path between the communicating nodes. It is the duration between the sending and the reception time, which are updated by the source and the destination, respectively. The Life time determines the maximum duration of a given flooding to reach the destination. Life time is continuously decremented until its expiration, and after that, all RREQs are dropped in order to make a restriction on the network flooding. The Source ID and Destination ID represent the identifiers of the communicating UAVs. The Previous ID field is updated at each intermediate UAV as the RREQ is flooded across the network. It represents the appropriate previous UAV in order to plot the routing path when the RREP is forwarded back to the source UAV. This field is stored locally in the routing table of each intermediate UAV in order to be used later during the data delivery. The Motion Information includes the mobility details (i.e., position, speed, and velocity) of each transited UAV and it is used to calculate the connectivity expiration time of the link $C E_{\overline{u_{i} u_{j}}}$ between each successive pair of UAVs $\overline{u_{i} u_{j}}$, and so on, until we get the minimum connectivity expiration time of the integral path $C E_{P}$. The connectivity expiration of the routing path is the time when any link between two successive UAVs breaks, and consequently, the current path between the communicating UAVs also breaks. The HOPs is the number of transited intermediate UAVs. The $R_{P}$ is the lowest residual energy of all succession of UAVs constituting the path. The Priority field can take different values according to the importance of the data packet or the application which belongs (i.e., 0 is the highest priority).

\begin{tabular}{|l|l|}
0 & 31 \\
\hline \multicolumn{3}{|c|}{ Communication ID } \\
\hline Delayp (s) & Life time \\
\hline \multicolumn{2}{|c|}{ Source ID } \\
\hline Destination ID \\
\hline Previous ID \\
\hline Motion information \\
\hline Priority \\
\hline HOPS \\
\hline
\end{tabular}

Fig. 3: RREQ packet format.

The RREP packet format is represented in Figure 4, where $C E_{P}$ and $R_{P}$ represent the Lowest connectivity expiration time (i.e., the weakest link of the path between two successive UAVs) and the residual energy of the selected path (i.e., the UAV with lowest remaining energy), respectively. In addition, the other fields are same as the fields included in the RREQ packet except for the Destination field. The latter contains both the position and the identifier of the destination, which are required during the maintenance process.

\begin{tabular}{|c|c|c|}
\hline \multicolumn{2}{|c|}{16} & 31 \\
\hline \multicolumn{3}{|c|}{ Communication ID } \\
\hline \multicolumn{3}{|c|}{ Source ID } \\
\hline \multicolumn{3}{|c|}{ Destination } \\
\hline \multicolumn{3}{|c|}{ Motion information } \\
\hline Delayp (s) & $C E_{P} \quad(s)$ & $R_{P}$ \\
\hline
\end{tabular}

Fig. 4: RREP packet format. 
Once the RREP packet is intercepted by the source UAV, the routing path is well traced and the data packet is generated and ready to be sent (c.f., Figure 5). The size of the data packet is set to $m$ bytes which can be modified according to the amount of information to be sent. To know if it is possible to send other data packets across the time (i.e., the current path and if it is still valid or not), a header which does not exceed 16 bytes containing nearly the same fields as in the RREP has to be merged with the useful data frame. These fields are permanently checked before each data transmission to make the right forwarding decision and avoid packet losses.

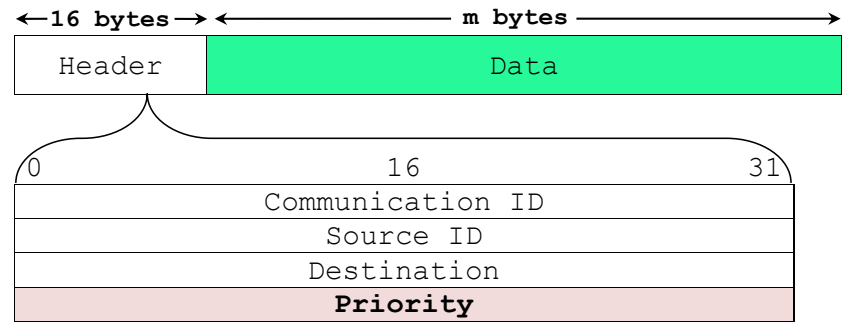

Fig. 5: Data packet format.

2) Link connectivity expiration: Based on the same method used in [35], the link connectivity expiration time $C E$ of any two UAVs deployed in a 3D scenario can be defined as the connectivity duration of the two UAVs within a fixed range Range. As shown in Figure 6, let $u_{1}$ and $u_{2}$ be two UAVs with a Line-of-Sight (LoS) range of Range, non zero speeds $V_{1}$ and $V_{2}$, their initial locations be $\left(X_{1}^{\prime}, Y_{1}^{\prime}, Z_{1}^{\prime}\right)$ and $\left(X_{2}^{\prime}, Y_{2}^{\prime}\right.$, $Z_{2}^{\prime}$ ), and their respective velocity angles $\theta_{1}, \phi_{1}$ and $\theta_{2}, \phi_{2}$.

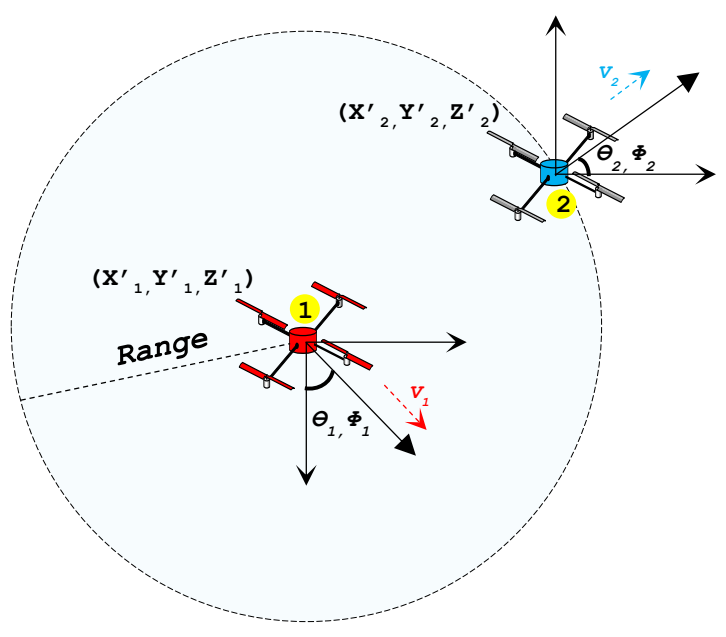

Fig. 6: Link connectivity expiration.

After a period of time $C E$, the distance $D$ between $u_{1}$ and $u_{2}$ can be calculated using the equation (2):

$$
\begin{gathered}
D^{2}=(i+l C E)^{2}+(j+m C E)^{2}+(k+n C E)^{2}, \\
C E^{2}\left(l^{2}+m^{2}+n^{2}\right)+C E(2 i l+2 j m+2 k n)+i^{2}+j^{2}+k^{2}=D^{2} .
\end{gathered}
$$

where,

$$
\begin{aligned}
i & =\left(X_{1}^{\prime}-X_{2}^{\prime}\right), \\
j & =\left(Y_{1}^{\prime}-Y_{2}^{\prime}\right), \\
k & =\left(Z_{1}^{\prime}-Z_{2}^{\prime}\right), \\
l & =\left(V_{1} \sin \theta_{1} \cos \phi_{1}-V_{2} \sin \theta_{2} \cos \phi_{2}\right), \\
m & =\left(V_{1} \sin \theta_{1} \sin \phi_{1}-V_{2} \sin \theta_{2} \sin \phi_{2}\right), \\
n & =\left(V_{1} \cos \theta_{1}-V_{2} \cos \theta_{2}\right) .
\end{aligned}
$$

To calculate connectivity expiration based on Range, we assume that the maximum distance where two UAVs can communicate is when $D=$ Range. For this purpose, we suppose that after a period of time $C E$, the distance between these two UAVs is the transmission range Range. The equation (2) becomes as follows:

$C E^{2}\left(l^{2}+m^{2}+n^{2}\right)+C E(2 i l+2 j m+2 k n)+i^{2}+j^{2}+k^{2}-\operatorname{Range}^{2}=0$.

The connectivity expiration time of the link between these two UAVs can be calculated based on the following equation (4):

$$
C E=\frac{-y \pm \sqrt{y^{2}-4 x z}}{2 x} .
$$

where,

$$
\begin{aligned}
& x=l^{2}+m^{2}+n^{2}, \\
& y=2 i l+2 j m+2 k n, \\
& z=i^{2}+j^{2}+k^{2}-\text { Range } e^{2} .
\end{aligned}
$$

Always based on Figure 6, we take the case when $u_{2}$ intercepts the RREQ packet broadcasted by $u_{1}$, it extracts the details included in the Motion Information field (i.e., velocity angles, speed, and position). Based on this information, it calculates $C E_{\overline{u_{1} u_{2}}}$ of the link $\overline{u_{1} u_{2}}$ that connects it with $u_{1}$ based on the equation (4). If the computed $C E_{\overline{u_{1} u_{2}}}$ is smaller than the $C E_{P}$ already included in the intercepted RREQ packet, $C E_{P}$ field is updated with the new value of $C E_{\overline{u_{1} u_{2}}}$, which is currently considered as the weakest link of the path. Otherwise, $u_{2}$ includes its own motion details, updates the other fields when it is needed and re-broadcasts the RREQ. This process is repeated until a routing path is built towards the target destination.

3) Energy consumption management: The wise management of remaining energy levels in UAVs is quite crucial for both the network lifetime and the reliable data delivery. Each UAV periodically calculates its ratio of energy remaining capacity $\left(R_{\text {erc }}\right)$ based on the following equation:

$$
R=R_{\text {erc }} \%=\frac{\text { Battery residual energy }}{\text { Battery full energy }} \times 100 .
$$

Three intervals of remaining energy levels are defined: (i) High energy level ( $R \geq 66 \%$ ), (ii) Medium energy level $(30 \% \leq R \leq 66 \%)$, and (iii) Low energy level $(R \leq 33 \%)$. If a UAV has a low energy level (i.e., below $\tau$ ), none of the packets will be forwarded and they are immediately dropped, except 
data packets with high priorities (i.e., Priority $=0$ ) or in the case where the UAV belongs to a path already established. Otherwise, upon receiving an RREQ packet, the UAV has to update the $R_{P}$ field with its $R$ value only when $\left(R_{P}>R\right)$.

For each UAV, Three distributions of energy consumption are assumed for our work (see Table III). First, in practice and according to [36], the energy consumption is dominated by the propulsion energy of UAVs. For our work, we assume that $70 \%$ is dedicated to the mobility of UAVs. Second, wireless communications with $25 \%$, because most of the time the consumption peaks are distinguished particularly during forwarding and receiving packets [34]. Finally, the consumption during idling is estimated at 5\% since UAVs continue making both the exchange of Hello packets and listen operations (not receiving data) while they are hovering the area. For simplicity, we ignore the UAV energy calculation and storage.

TABLE III: Distributions of energy consumption in the UAV.

\begin{tabular}{|l|c|}
\cline { 2 - 2 } \multicolumn{1}{c|}{} & Energy consumption \\
\hline Propulsion energy & $70 \%$ \\
\hline Wireless communications & $25 \%$ \\
\hline Idle state & $5 \%$ \\
\hline
\end{tabular}

\section{B. Discovery process}

To illustrate all the processes of $\mathrm{ECaD}$, let us consider the network in Figure 7 . When $u_{S}$ wants to establish a low priority communication with $u_{D}$, it initiates a route discovery (similar to AODV [18]) in the case when there is no connected path available in its routing table. An RREQ packet is generated and broadcasted across the network in order to establish a routing path towards $u_{D}$. While crossing the network, the RREQ packet records a set of information, which determines the connectedness of routing paths while taking into account the balanced energy consumption among the UAVs. To reduce or to avoid the broadcast storm, the UAV checks whether it has received the same RREQ packet or not based on the Communication ID field. If, for instance, a received RREQ packet has the same Communication ID with a previously received one, it will be automatically dropped. Otherwise, some fields are updated, some others are cached in the current UAV's routing table, and then, the RREQ packet is re-broadcasted. As an illustration, a set of routing tables is depicted in Figure 8.

A set of routing decisions $(R D s)$ can be taken at different levels according to the network to designate the appropriate previous hops in order to trace the different possible paths between the communicating UAVs. As seen in Figure 7, four levels of RDs are carried out as follows:

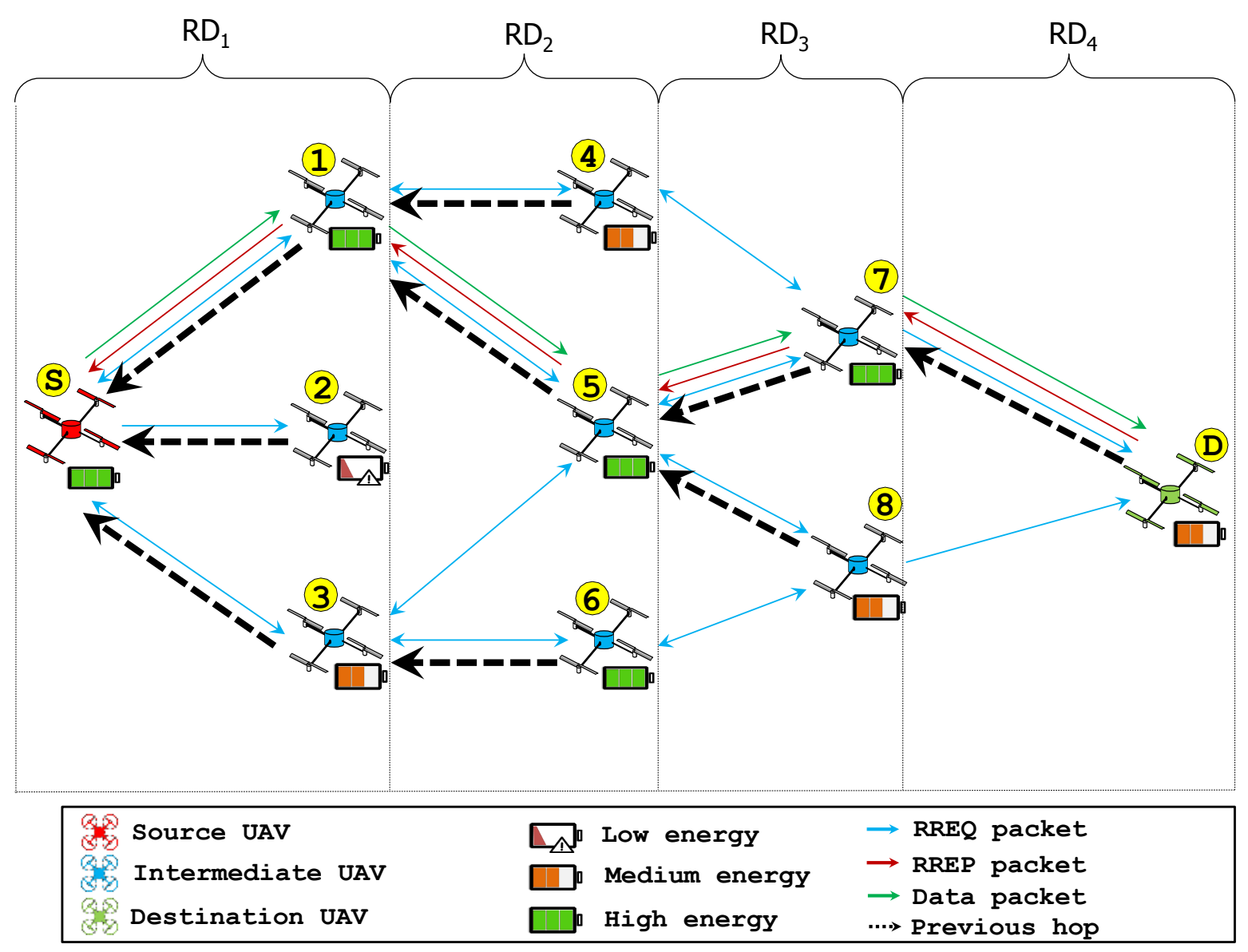

Fig. 7: Principle of flooding functioning. 
1) $R D_{1}: u_{S}$ generates an RREQ packet that contains useful information, such as the Communication ID, the identifiers of the communicating UAVs (source and destination), and its motion information. Then, the RREQ is broadcasted to all its neighbors $N\left(u_{S}\right)=\left\{u_{1}, u_{2}, u_{3}\right\}$. The same routing entry is added in the routing tables of all $N\left(u_{S}\right)$ as soon as they receive the RREQ broadcasted by $u_{S}$, which indicates the previous hop $u_{S}$. Moreover, as for $u_{S}$, since it has no previous hop, it adds a different routing entry in its routing table indicating the identifiers of the destination and the communication (see fields highlighted in blue in Figures 8(a) and 8(b)). The values of certain fields are copied directly to their routing tables, such as the destination identifier, the Communication ID, and the broadcaster is initially identified as the previous hop. Each $u_{i} \in N\left(u_{S}\right)$ generates an RREQ packet in which the $H O P s$ value is incremented, $R_{P}=\min \left(R_{u_{S}}, R_{u_{i}}\right)$, and $C E_{P}=C E_{\overline{u_{S} u_{i}}}$. All this information is included in the newly generated RREQ packet, and then re-broadcasted. It is worthy to mention that $u_{2}$ in range only of $u_{S}$ and it cannot make the broadcast, since its battery is in low level $\left(R_{u_{2}}<\tau\right)$ and it can only receive packets. Moreover, $u_{S}$ drops all RREQs broadcasted by $u_{1}$ and $u_{3}$. This is because $u_{S}$ has found itself as the designated previous hop of $u_{1}$ and $u_{3}$ and it has already a routing entry of the intercepted RREQs.

2) $R D_{2}: u_{4}$ and $u_{6}$ intercept only one RREQ from $u_{1}$ and $u_{3}$, respectively. In this case, there is no routing decision to take and the broadcasters are directly elected as their previous hops. However, as for $u_{5}$ that has intercepted two RREQs, a routing decision has to be made to define the adequate previous UAV. If we suppose that $u_{5}$ initially intercepts the RREQ of $u_{3}$, it adds a new routing entry and designates $u_{3}$ as its previous hop. As for the second intercepted RREQ from $u_{1}, u_{5}$ finds that it has both a routing entry with the same Communication ID and it is not its previous hop. Consequently, the previous hop in its routing table has to be re-calculated based on a multi- criteria score using the following equation (6) for each previous hop:

$$
S_{\overline{u_{i} u_{j}}}=\frac{R_{P}}{H O P S} \times\left\lfloor\frac{C E_{P}}{\text { DelayP }}\right\rfloor .
$$

Note, that from the equation (6), we notice the following remarks:

- $u_{i}$ is the current UAV, while $u_{j}$ is the broadcaster of the RREQ. Delay $y_{P}=\operatorname{Delay}_{P_{i}\left(u_{S}, u_{i}\right)}$ which is the required time for a packet to transit the path from $u_{S}$ until $u_{i}$ via $u_{j}$.

- $R_{P}=\min \left(R_{P_{i}\left(u_{S}, u_{j}\right)}, R_{u_{i}}\right)$ and $C E_{P}=$ $\min \left(C E_{P_{i}\left(u_{S}, u_{j}\right)}, C E_{\overline{u_{i} u_{j}}}\right)$, which represent the residual energy and the connectivity expiration of the path from the source $u_{S}$ through a given previous hop $u_{j}$ until the current UAV $u_{i}$, respectively.

- The floor of $\left\lfloor\frac{C E_{P}}{\text { Delay }_{P}}\right\rfloor$ is a scalar that represents whether the routing path through a given previous hop still remains connected or not during the data delivery. Therefore, it grows only in the positive side and can $\left\lfloor\frac{C E_{P}}{\text { Delay }_{P}}\right\rfloor=0$ only when $C E_{P}<$ Delay $_{P}$ or $C E_{P}=0$, which means that the path is weakly connected during the data delivery and it can be disconnected at any time. However, if $\left\lfloor\frac{C E_{P}}{\text { Delay }_{P}}\right\rfloor>0$, it means that there is a high probability that this routing path through a given previous hop remains connected during the data delivery.

- $S_{\overline{u_{i} u_{j}}}$ is the score of the full path until the current $u_{i}$ through the previous hop $u_{j}$.

- $S_{\overline{u_{i} u_{j}}}$ has a proportional relationship with $R_{P}$ and $C E_{P}$ which are progressively calculated until the current UAV during the discovery process.

- Previous hops with high scores are preferable because they do not belong to weakly connected paths which can be composed of UAVs having low energy levels.

\begin{tabular}{|c|c|c|c|c|c|}
\hline \multicolumn{7}{|c|}{ Routing Table $\left(u_{1}\right)$} \\
\hline Prev. hop & Next & Destination & Com. ID & $C E_{\overline{u_{1} u_{5}}}(\mathrm{~s})$ & $R_{u_{5}}$ \\
\hline Us $_{\mathrm{s}}$ & $\mathrm{U}_{5}$ & UD & 01 & $6(\mathrm{~s})$ & High \\
\hline$\ldots$ & $\ldots$ & $\ldots$ & $\ldots$ & $\ldots$ & $\ldots$ \\
\hline
\end{tabular}

(b) Routing table of $u_{1}$.

\begin{tabular}{|c|c|c|c|c|c|}
\hline \multicolumn{7}{|c|}{ Routing Table $\left(\mathrm{u}_{7}\right)$} \\
\hline Prev. hop & Next & Destination & Com. ID & $C E_{\overline{u_{7} u_{D}}}(\mathrm{~s})$ & $R_{u_{D}}$ \\
\hline $\mathrm{u}_{5}$ & $\mathrm{u}_{7}$ & $\mathrm{u}_{\mathrm{D}}$ & 01 & $6,5(\mathrm{~s})$ & Medium \\
\hline$\ldots$ & $\cdots$ & $\cdots$ & $\cdots$ & $\cdots$ & $\cdots$ \\
\hline
\end{tabular}

(d) Routing table of $u_{7}$.

\begin{tabular}{|c|c|c|c|c|c|}
\hline \multicolumn{7}{|c|}{ Routing Table (u $\left.\mathrm{u}_{\mathrm{D}}\right)$} \\
\hline Prev. hop & Next & Destination & Com. ID & $C E(\mathrm{~s})$ & $R$ \\
\hline $\mathrm{u}_{7}$ & - & $\mathrm{uD}_{\mathrm{D}}$ & 01 & - & - \\
\hline$\cdots$ & $\cdots$ & $\cdots$ & $\cdots$ & $\cdots$ & $\cdots$ \\
\hline
\end{tabular}

(f) Routing table of $u_{D}$.

(e) Routing table of $u_{8}$.

Fig. 8: Routing tables of UAVs. 
In our case, $u_{1}$ obtains the best score and it is designated as the previous hop of $u_{5}$. Then, $u_{4}, u_{5}$, and $u_{6}$ update all required information in their newly generated RREQs and broadcast them to all their neighbors.

3) $R D_{3}$ : two routing decisions are made at this level. Indeed, both $u_{7}$ and $u_{8}$ have designated $u_{5}$ as their previous hop using the same method as in $R D_{2}$.

4) $R D_{4}$ : the destination $u_{D}$ makes the final routing decision to designate the last previous hop, and thus plotting the full and single path that exists between the communicating UAVs. $u_{7}$ is designated as the previous hop of $u_{D}$ and this information is added in the routing table of $u_{D}$ (c.f., Figure 8(f)).

As a general rule, all UAVs having at least two previous UAVs calculate a score for each previous UAV in order to select the most adequate previous one. Table IV shows the different routing decisions made during the transition of the RREQ packet across the network shown in Figure 7. This can help to efficiently exploit the network lifetime and connectedness and to avoid critical situations where routing paths are weakly connected and do not achieve balanced energy consumption among UAVs. From Table IV, the suitable routing path can be deducted based on the sequence of previous hops until the source $u_{S}$. Indeed, the destination $u_{D}$ receives two different RREQ packets from $u_{7}$ and $u_{8}$ representing two different paths. Consequently, a routing decision has to be made to select the most appropriate previous hop. The destination selects $u_{7}$ as its previous hop, and as a result, a valid route is established between $u_{S}$ and $u_{D}$.

The RREQ packet will be handled by all nodes as shown in Algorithm 1:

An RREP packet is generated and unicastly sent back to the source through the sequence of the selected previous hops. At each hop of the RREP packet, a set of updates is made to the routing entry already added at each transited UAV during the flooding of the RREQ packet (fields highlighted in red in Figure 8). As shown in Figure 8(d), when $u_{7}$ intercepts the RREP packet, it adds the sender (destination) as the next hop, the connectivity expiration of the link $C E_{\overline{u_{7} u_{D}}}$, and the residual energy $R_{u_{D}}$ of the destination (i.e., $u_{D}$ periodically shares $R_{u_{D}}$ through Hello packets). This can help to predict with a high accuracy a disconnection or a battery failure of each link composing the full routing path. $C E_{P}$ and $R_{P}$ are also re-calculated, which allows both the source and intermediate UAVs to have an idea of the routing path validity and if it is possible to make other data transmission across the time.

The RREP packet will be handled by each transited UAV as shown in Algorithm 2:

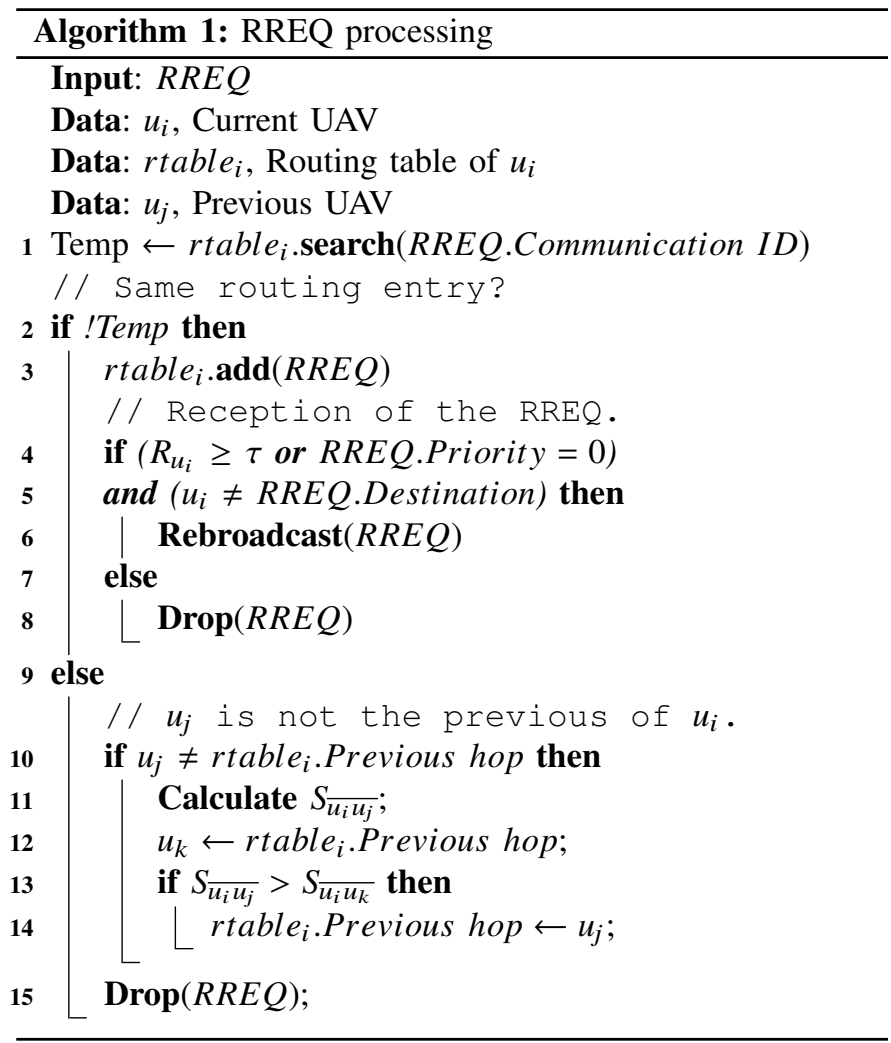

TABLE IV: Routing decisions during the discovery process.

\begin{tabular}{|l|c|c|c|c||c|c||c|c|}
\cline { 2 - 10 } \multicolumn{1}{c|}{} & \multicolumn{8}{c|}{ UAVs making selection of their previous hops } \\
\cline { 2 - 11 } \multicolumn{1}{c|}{} & \multicolumn{2}{c|}{$u_{5}$} & \multicolumn{2}{c|}{$u_{7}$} & \multicolumn{2}{c|}{$u_{8}$} & \multicolumn{2}{c|}{$u_{D}$} \\
\hline Prev. hops & $u_{1}$ & $u_{3}$ & $u_{4}$ & $u_{5}$ & $u_{5}$ & $u_{6}$ & $u_{7}$ & $u_{8}$ \\
\hline$C E_{P}(s)$ & 6 & 4 & 5 & 6 & 6 & 4 & 6 & 5 \\
\hline$R_{P}$ & 80 & 50 & 40 & 80 & 80 & 50 & 60 & 50 \\
\hline Delay $(s)$ & 0.3 & 0.3 & 0.45 & 0.45 & 0.45 & 0.45 & 0.6 & 0.6 \\
\hline HOPs & 2 & 2 & 3 & 3 & 3 & 3 & 4 & 4 \\
\hline$S_{\overline{u_{i} u_{j}}}$ & 800.00 & 333.33 & 296.29 & 355.55 & 355.55 & 148.14 & 150 & 104.16 \\
\hline
\end{tabular}




\section{Data Delivery process}

After receiving the RREP packet, the source can start the data transmission through its next hop and the rest of the transition is carried out automatically until the target destination (see Figure 7). The routing tables are checked before each data transmission since the information of the routing tables can be updated at any time. Indeed, the energy consumption is different in each UAV, which indeed requires a permanent update of the routing tables (i.e., the $R$ field) based on the periodical exchange of Hello packets containing fresh information. This is useful to avoid the UAV with a low battery level and to find other alternative solutions. However, there is an exception for data packets in which their priorities are high, particularly those related to real-time applications where UAVs with low battery levels are tolerated to continue the transition process of data packets.

To illustrate the data delivery process, we always refer to Figure 7. We note that every time a UAV receives a data packet, it first checks its updated routing table. If, for instance, $u_{5}$ receives a data packet destined for $u_{D}$, it first checks its routing routing table (c.f., Figure $8(\mathrm{c})$ ). After this verification, $u_{5}$ finds that $C E_{\overline{u_{5} u_{7}}}$ is not yet expired and $R_{u_{7}}$ of the next hop has a high energy level. Consequently, $u_{5}$ forwards directly the data packet to $u_{7}$ where the same process is repeated until the data packet will be delivered to $u_{D}$.

\section{Maintenance Process}

Several causes can be identified behind a disconnection, such as the high mobility of UAVs or a UAV failure due to its limited energy capacity. In this case, the current UAV can detect immediately this link failure based on $C E$ of this link, thus avoiding data packet losses. Consequently, the routing entry is considered to be not valid and has to be purged from the routing table. To find other alternative solutions, the current UAV checks its table of neighbors to find the suitable forwarder. In a general case, the closest neighbor to the target destination is selected to continue transiting the data packet. Nevertheless, another factor has to be taken into account which is the residual energy $R$ of each neighbor UAV. If the priority of the data packet is high, the geographically closest UAV to the target destination is selected even if its battery is in low level. Otherwise, only neighbor UAVs with at least medium energy levels are considered to select among them the closest one to the destination. When the network is poorly dense and there is no neighbor, an RERR packet is sent back to the source to re-initiate the discovery process to find other connected routing paths.

To provide a clear explanation, a concrete illustration is given in Figure 10 by zooming in Figure 7 . We remark that the already established routing path was disconnected at $\overline{u_{5} u_{7}}$. Two different cases can be distinguished, (i) A low priority data packet and (ii) A high priority data packet. In the first

\begin{tabular}{|c|c|c|c|c|c|}
\hline \multicolumn{7}{|c|}{ Routing Table (u) } \\
\hline Prev. hop & Next & Destination & Com. ID & $C E_{\overline{u_{5} u_{8}}}(\mathrm{~s})$ & $R_{u_{8}}$ \\
\hline $\mathrm{u}_{1}$ & $\mathrm{u}_{8}$ & $\mathrm{uD}_{\mathrm{D}}$ & 01 & $1(\mathrm{~s})$ & Low \\
\hline$\ldots$ & $\ldots$ & $\ldots$ & $\ldots$ & $\ldots$ & $\ldots$ \\
\hline
\end{tabular}

(a) Routing table of $u_{5}$.

\begin{tabular}{|c|c|c|c|c|c|}
\hline \multicolumn{6}{|c|}{ Routing Table $\left(\mathrm{u}_{8}\right)$} \\
\hline Prev. hop & Next & Destination & Com. ID & $C E_{\overline{u_{8} u_{D}}}(\mathrm{~s})$ & $R_{u_{D}}$ \\
\hline$u_{5}$ & UD & UD & 01 & $2(\mathrm{~s})$ & Medium \\
\hline$\ldots$ & $\cdots$ & $\ldots$ & $\ldots$ & $\ldots$ & $\cdots$ \\
\hline
\end{tabular}

(b) Routing table of $u_{8}$.

Fig. 9: Routing tables of UAVs in the maintenance process.

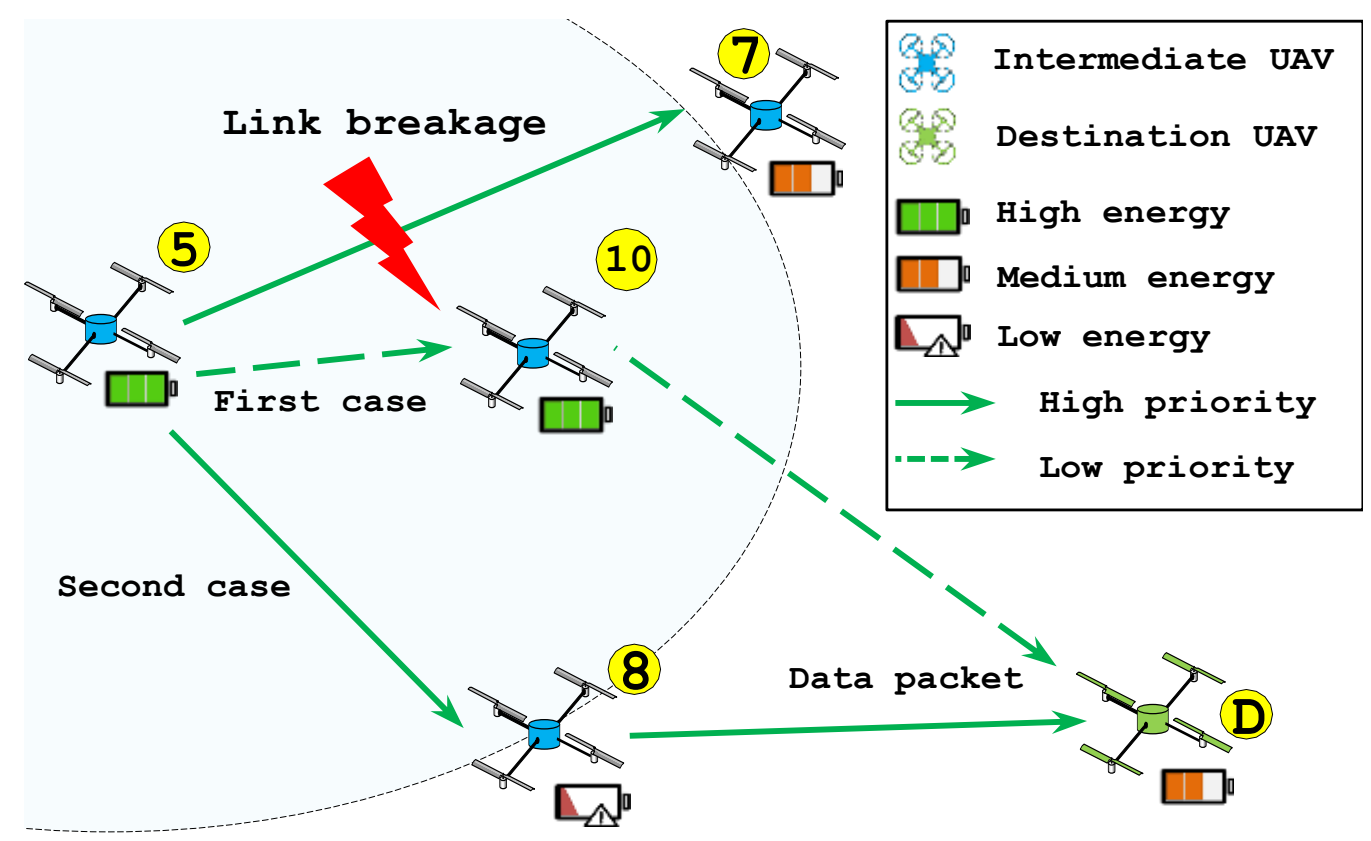

Fig. 10: Path failure recovery. 
case, $u_{5}$ checks its updated table of neighbors to find, if possible, the closest neighbor UAV to $u_{D}$ where preferably its battery is not in low level. As a result, $u_{10}$ is the adequate forwarder responding to the aforementioned condition (i.e., $u_{10}$ is a newly introduced UAV in the network). This process is repeated until the data packet reaches $u_{D}$. In the second case, $u_{5}$ selects the closest neighbor to $u_{D}$ regardless of its energy level. $u_{8}$ is found as the next forwarder where the data packet is transmitted, and so on until the data packet will be delivered to the target destination. This new transition results in a set of modifications in the routing tables of $u_{5}$ and $u_{8}$ (c.f., Figure 9). Indeed, $u_{5}$ and $u_{8}$ modify their next hops to $u_{8}$ and $u_{D}$, respectively, and include their residual energy levels as well as their connectivity expiration times as shown in Figures 9(a) and 9 (b).

In conclusion, this method is only considered to supplement unexpected path failures because, in most cases, the primary established path has both the longest connectivity expiration and the longest lifetime duration. Hence, before the expiration of this primary path, data packets can be preserved from the loss by finding other alternative solutions. Effectively, they are not more appropriate, but better than the re-initialization of the discovery process. All data packets are handled according to Algorithm 3.

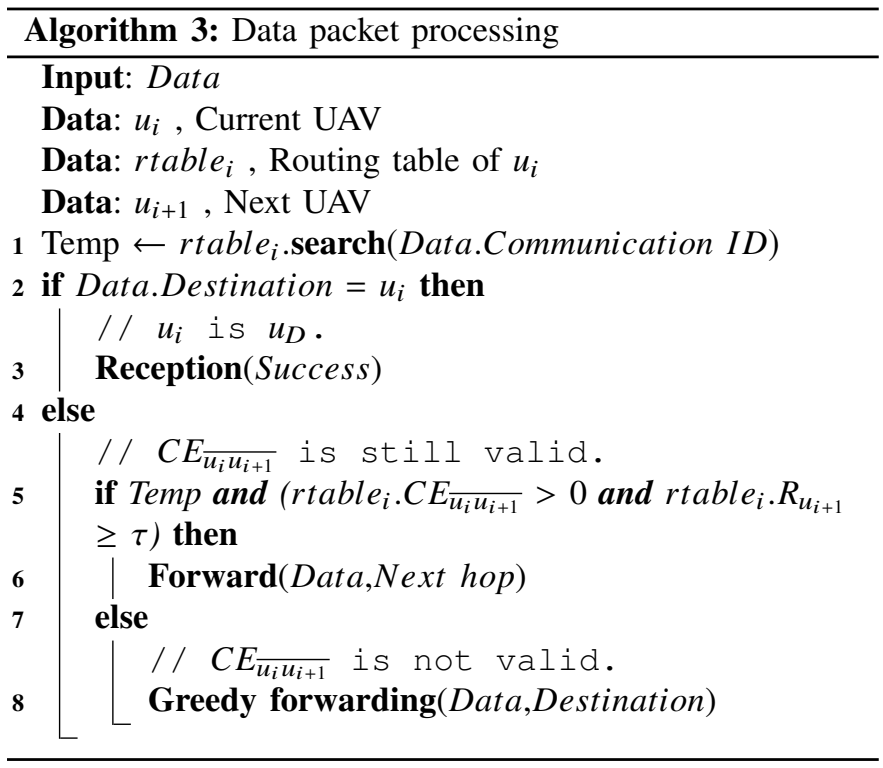

Thus far, ECaD considers multiple routing processes which can be executed simultaneously, and especially when there are many pairs of communicating UAVs. Consequently, we have to be sure that no routing loop occurs during the functioning of ECaD. Moreover, since we use an on-demand technique, the amount of packets and the elapsed time exploited over a communication session is variable from one case to another. This is why we need to analyze the different complexities of $\mathrm{ECaD}$ in worst cases.

\section{TheOretical ANALYsis of ECAD}

In this section, we prove the ECaD's loop freedom property and we estimate its costs in terms of the amount of exchanged packets and time complexities. To this aim, we assume that $U=\left\{u_{1}, u_{2}, \ldots, u_{n}\right\}$ is a finite set of $n$ UAVs.

Theorem 1. ECaD is loop free.

Proof. We prove it by contradiction. Let us suppose that there exists a loop in the selected routing path, where $u_{i}, u_{i+1}$, and $u_{i+2}$ are in the circle, and the packets are forwarded from $u_{i+2}$ to $u_{i+1}, u_{i+1}$ to $u_{i}$, and then are routed back to $u_{i+2}$ (c.f., Figure 11).

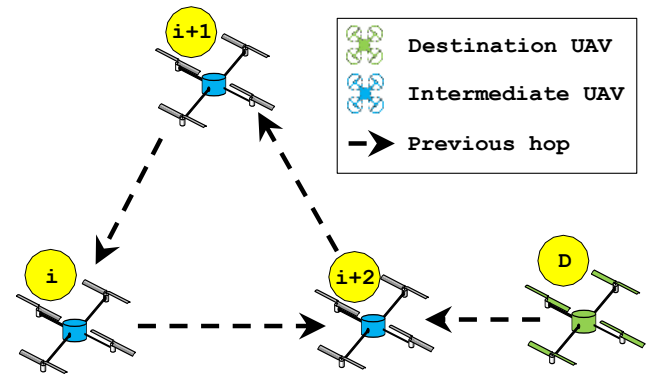

Fig. 11: A loop scenario.

Suppose that the source is $u_{S}$ and according to the routing algorithm of $\mathrm{ECaD}$, a score is calculated for each link between UAVs forming the circle:

$$
\begin{aligned}
S_{\overline{u_{i} u_{i+2}}} & =\frac{R_{P_{i}}}{H O P S} \times\left\lfloor\frac{C E_{P_{i}}}{\text { Delay } y_{P_{i}}}\right\rfloor \\
S_{\overline{u_{i+1} u_{i}}} & =\frac{R_{P_{i+1}}}{H O P s+1} \times\left\lfloor\frac{C E_{P_{i+1}}}{\text { Delay } y_{P_{i+1}}}\right\rfloor \\
S_{\overline{u_{i+2} u_{i+1}}} & =\frac{R_{P_{i+2}}}{H O P s+2} \times\left\lfloor\frac{C E_{P_{i+2}}}{\text { Delay } y_{P_{i+2}}}\right\rfloor .
\end{aligned}
$$

where,

$$
\begin{aligned}
R_{P_{i}} & =R_{P_{i+1}}=R_{P_{i+2}}=\min \left(R_{P_{i}\left(u_{S}, u_{i+2}\right)}, R_{u_{i}}\right), \\
C E_{P_{i}} & =C E_{P_{i+1}}=C E_{P_{i+2}}=\min \left(C E_{P_{i}\left(u_{S}, u_{i+2}\right)}, C E_{\overline{u_{i} u_{i+2}}}\right), \\
\operatorname{Delay}_{P_{i}} & =\operatorname{Delay}_{P_{i}\left(u_{S}, u_{i}\right)}, \\
\operatorname{Delay}_{P_{i+1}} & =\operatorname{Delay}_{P_{i}}+\operatorname{Delay}_{\overline{u_{i} u_{i+1}}}, \\
\operatorname{Delay}_{P_{i+2}} & =\text { Delay }_{P_{i+1}}+\operatorname{Delay}_{\overline{u_{i+1} u_{i+2}}}, \\
\operatorname{Delay}_{P_{i}} & <\operatorname{Delay}_{P_{i+1}}<\operatorname{Delay}_{P_{i+2}}, \\
\text { HOPs } & <\text { HOPs }+1<\text { HOPs }+2,
\end{aligned}
$$

Consequently, we deduct that:

$$
S_{\overline{u_{i} u_{i+2}}}>S_{\overline{u_{i+1} u_{i}}}>S_{\overline{u_{i+2} u_{i+1}}} \text {. }
$$

And so that $u_{i+2}$ be the previous of $u_{i}$ it is necessary that:

$$
S_{\overline{u_{i+2} u_{i+1}}}>S_{\overline{u_{i} u_{i+2}}} \text {. }
$$

Which is a contradiction, and thus ECaD is loop free.

Lemma 1. (Upper boundary of ECaD time complexity). The time complexity is the sum of the time complexities of the $R R E Q$ flooding, the RREP forwarding, and the data delivery.

$$
T_{E C a D}=T_{R R E Q} \times 3
$$


where,

$$
\begin{aligned}
T_{R R E Q} & =N_{\text {Hops }} \times \text { Delay } \overline{\overline{u_{i} u_{i+1}}} \\
N_{\text {Hops }} & =\left\lfloor\frac{x}{\text { Range }}\right\rfloor
\end{aligned}
$$

Proof. The execution time of $\mathrm{ECaD}$ depends strongly on the diameter of the network, where the diameter is the maximum number of hops between any two UAVs. During the discovery process and in the worst case, we notice that the RREQ can be continuously flooded over the network until it reaches the target destination by transiting a given distance $x$. Indeed, $T_{R R E Q}$ in the worst case is the longest succession of hops in which the distance between any two consecutive hops is equal to the communication range Range. Based on these two parameters, it is easy to extract the required number hops $N_{\text {Hops }}$ between any communicating UAVs. It can be calculated by taking the floor of the division between the total distance and the communication range of UAVs. We assume that the estimated delay between each pair of successive UAVs $\left(u_{i}, u_{i+1} \in U\right)$ is Delay $\overline{\overline{u_{i} u_{i+1}}}$. Consequently, the transition time of the RREQ, the RREP, and the data is the sum of intermediate times of all transited UAVs multiplied by 3 . Thus, the $\mathrm{ECaD}$ time complexity is given in Equation (7). It should be stressed that in the general case, the selected hops can be any neighbor within range (i.e. with a distance less than the communication range) which would be beneficial for the performance compared to the worst scenario.

Lemma 2. (Upper boundary of ECaD messages' complexity). The messages' complexity is the sum of the time complexities of the RREQ flooding, the RREP forwarding, and the data delivery.

$$
M_{E C a D}=2 \times b+(n-1) .
$$

where,

$$
b=\left\lfloor\frac{x}{\text { Range }}\right\rfloor+1
$$

Proof. The number of exchanged messages of ECaD depends on the number of nodes. When there is no path failure and in the worst case, the number of exchanged messages is equal to the number of all routing packets exchanged during the discovery process and the transition of the data packet. The maximum number of exchanged RREQs is where each UAV except the destination broadcasts and receives an RREQ from all UAVs of the network and it is equal to $n-1$. It should be stressed that the general case depends on the distance between the communicating nodes $\left(u_{S}\right.$ and $\left.u_{D}\right)$. The path between the source and destination would comprise $b=\left\lfloor\frac{x}{\text { Range }}\right\rfloor+1$ messages. To that effect, each of the RREP and the data require $b$ messages. Regarding the RREQ messages, since we have considered a basic broadcast of RREQs, the number of exchanged messages always remains $n-1$.
As for the computation overhead, in the worst case, the calculation of the score mentioned in Equation (6) can be carried out $n-3$ times, which is explained by the received number of RREQs that can get each UAV during single data transmission. If we consider that a network comprises $n \mathrm{UAVs}$, the complexity of computation overhead in the whole network can be expressed as $O\left(n^{2}\right)$.

Similarly, the complexity of route discovering process is based on a basic physical broadcast technique where each UAV in the network (except the destination) has to rebroadcast each received RREQ packet, that is a complexity of $O(n)$. If we consider the whole network as a single system, that is a complexity of $O\left(n^{2}\right)$.

\section{Performance Evaluation}

A set of experiments is carried out in NS-2 which is supported by MobiSim [37] to generate the movements of UAVs. Indeed, a Random Way Point (RWP) mobility model is adopted for up to $100 \mathrm{UAVs}$, which are fairly distributed in a region of $2 \times 2 \times 1 \mathrm{~km}^{3}$ for 300 (s) of simulated time (c.f., Figure 13).

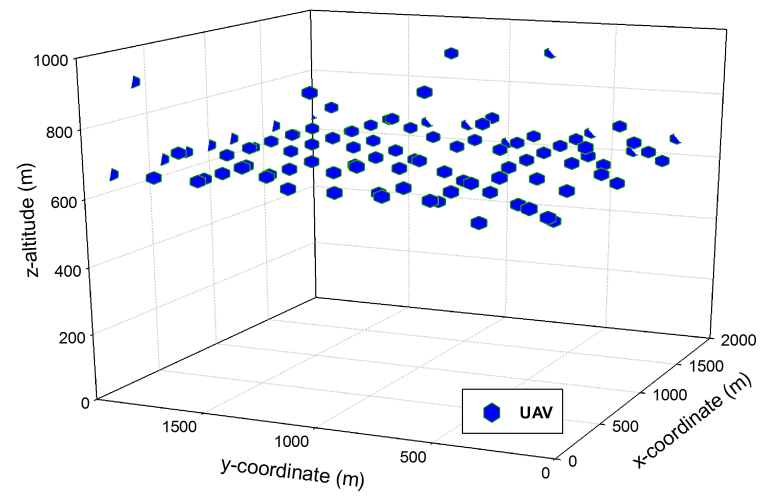

Fig. 13: Simulation scenario.

We suppose that the altitude of all UAVs is dynamic and does not exceed 1000 meters during the flight. It is worthy to mention that even if the RWP mobility model is not quite suitable for FANETs [38], it is used in our simulation to investigate the critical impact of random movements on routing protocols. For each UAV, the energy consumption is based on the distribution assumed in Table III. Several evaluation metrics are calculated, such as the ratio of energy remaining capacity $\left(R_{e r c}\right)$, the packet delivery ratio $(P D R)$, the end-to-end delay $(E E D)$, the overhead $(O H)$, and the Path stability under different speeds and densities. UVAR$S$ [20], MPGR [22], LEPR [24], CBLADSR [25], and the proposed $\mathrm{ECaD}$ are all evaluated and compared. Ten pairs of UAVs are randomly selected to communicate with each other. The tables of neighbors, as well as the routing tables, are updated using the periodical exchange of Hello packets (Hello interval $\approx 0.1(\mathrm{~s})$ ) and are purged after $10(\mathrm{~s})$ of inactivity. Each point in the obtained results depicts the mean of 50 runs with a confidence interval of $95 \%$. The rest of the parameter settings for simulations are shown in Table V. 
TABLE V: Simulation parameters.

\begin{tabular}{|c|c|c|}
\hline & Parameter & Value \\
\hline \multirow{6}{*}{ 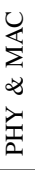 } & Frequency Band & $5 \mathrm{GHz}$ \\
\hline & Transmit power & $21.5 \mathrm{dBm}$ \\
\hline & Sensitivity & $-81.5 \mathrm{dBm}$ \\
\hline & Path loss model & Free-space \\
\hline & PHY model & IEEE $802.11 \mathrm{a}$ \\
\hline & Bitrate & $6 \mathrm{Mbit} / \mathrm{s}$ \\
\hline \multirow{5}{*}{ 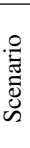 } & Area size & $2 \times 2 \mathrm{~km}^{2}$ \\
\hline & Simulation time & $300 \mathrm{~s}$ \\
\hline & Number of UAVs & {$[10,100]$} \\
\hline & Speed range & {$[3,30] \mathrm{m} / \mathrm{s}$} \\
\hline & Mobility generator & MobiSim [37] \\
\hline \multirow{5}{*}{ 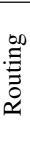 } & Mobility model & RWP \\
\hline & Communication range & $\approx 300$ \\
\hline & Data size & $1 \mathrm{~KB}$ \\
\hline & Initial energy available & $2000 \mathrm{~J}$ \\
\hline & Evaluation metrics & ility, and $\mathrm{OH}$ \\
\hline
\end{tabular}

\section{A. Ratio of energy remaining capacity $\left(R_{\text {erc }}\right)$}

Initially, we investigate the contour of residual energy levels of 49 UAVs that are uniformly deployed over the selected area. This is carried out with all evaluated routing protocols at the end of the simulation. The graphs depicted in Figure 12 have been smoothed using simple interpolation. The $\mathrm{x}$ axis and y-axis represent $2000 \mathrm{~m} \times 2000 \mathrm{~m}$ of a simulated area in which the $\mathrm{x}$-axis and $\mathrm{y}$-axis represent the $\mathrm{x}$-coordinates and y-coordinates of the deployed 49 UAVs. It is clearly shown in Figure 12(a) that ECaD achieves a well-balanced energy consumption across all UAVs. Indeed, for each data transmission, $\mathrm{ECaD}$ always selects the energy-rich routing path according to the priority of data packets to be sent. Moreover, even when the current path breaks, $\mathrm{ECaD}$ forwards data packets to the UAV where its $R$ is above $\tau$, thus avoiding
UAVs with low $R$ levels. However, in UVAR-S (c.f., Figure 12(b)), the routing paths are built based only on their degree of connectedness while neglecting the remaining energy level in each transited UAV, causing the consumption of more energy of certain UAVs than others.

We also notice the unbalanced energy consumption among the UAVs in LEPR, CBLADSR, and MPGR as shown in Figures 12(c), 12(d), and 12(e), respectively. Firstly, because the discovery process of LEPR is based on a topology-based routing protocol which is inadequate for highly dynamic networks, such as FANETs resulting in frequent disconnections, and consequently, multiple route discoveries consuming more energy. Secondly, in CBLADSR, the energy consumption is practically unbalanced between the $\mathrm{CHs}$ and their members, which is due to the inter-cluster communications always transiting through $\mathrm{CHs}$, thus consuming more energy. Finally, as MPGR selects the geographically closest UAV to the destination, it can sometimes happen that the same succession of UAVs is selected for more than one data transmission, and particularly when the UAVs move in the same direction. As a result, some UAVs consume less energy while others consume much more, which can provoke an early death of certain UAVs leading to a general breakdown of the network.

\section{B. Packet Delivery Ratio (PDR)}

As Figures 14(a) and 14(d) show, ECaD increased the PDR by almost half compared to UVAR-S, LEPR, and MPGR and by more than $10 \%$ compared to CBLADSR. This is explained by the gradual number of the discovered paths between the communicating UAVs, which can also guarantee a constant

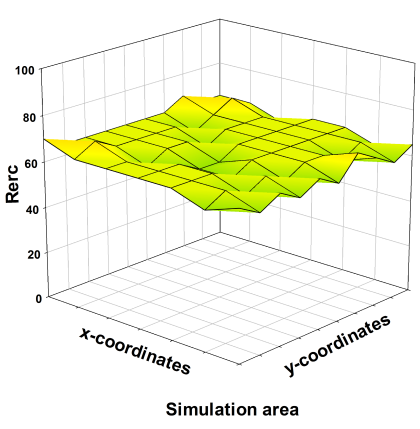

(a) $R_{\text {erc }}$ of ECaD.

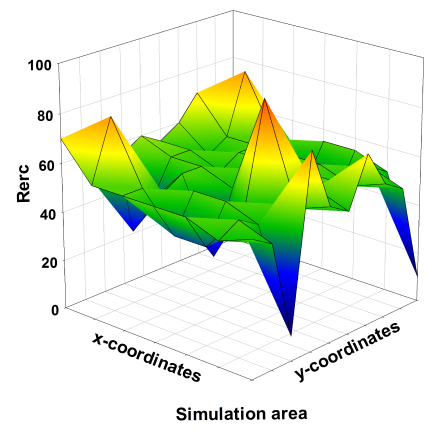

(b) $R_{\text {erc }}$ of UVAR-S.

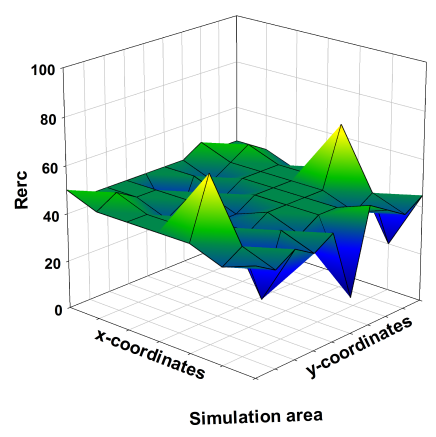

(c) $R_{e r c}$ of LEPR.

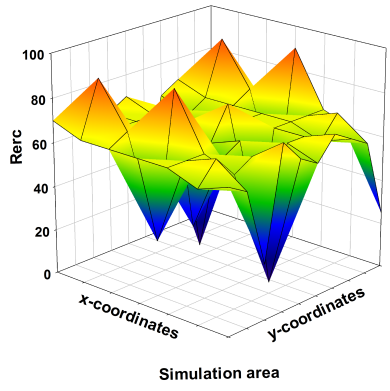

(d) $R_{\text {erc }}$ of CBLADSR.

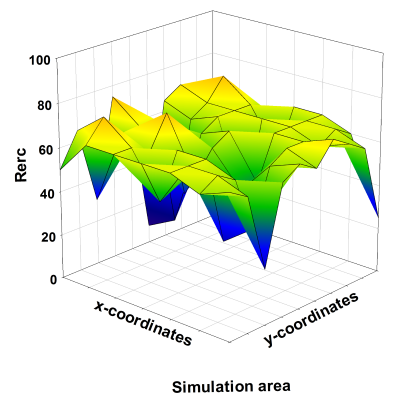

(e) $R_{\text {erc }}$ of MPGR.

Fig. 12: Contours of residual energy levels of $100 \mathrm{UAVs}$ at the end of the simulation 


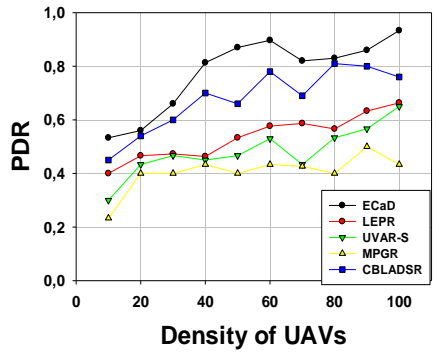

(a) PDR.

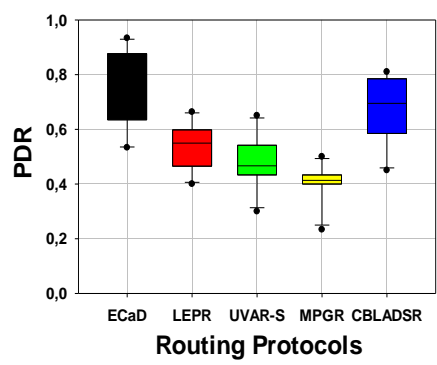

(d) Average PDR.

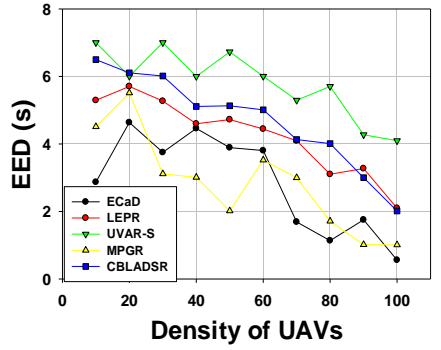

(b) EED.

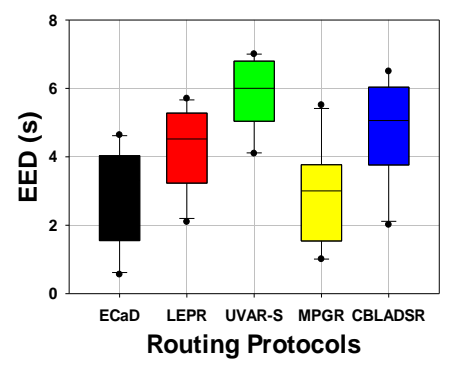

(e) Average EED.

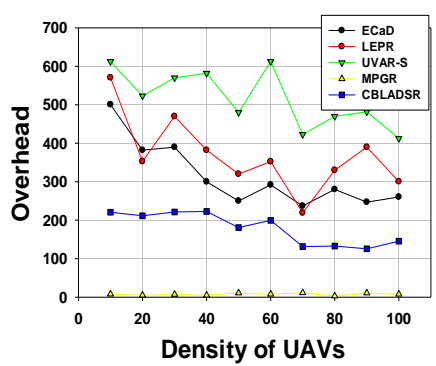

(c) Overhead.

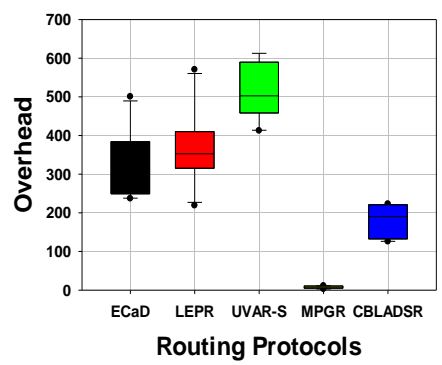

(f) Average Overhead.

Fig. 14: Simulation results vs. Density of UAVs $($ Speed $=30 \mathrm{~m} / \mathrm{s})$.

increase of alternative solutions in the case of path failures. In addition, the mechanism of the link expiration in terms of both energy and connectivity can provide reliable long lifetime routing paths for more data packets to send. However, CBLADSR neglects the link connectivity, and especially between the $\mathrm{CHs}$ even if they assume long communication ranges, causing frequent disconnections and several packet losses. For the other protocols, the packet losses are high and are directly related to the unbalanced energy consumption among the UAVs, where the data packets can be suddenly lost at an intermediate UAV having an insufficient energy level to continue the data transmission.

\section{End-to-End Delay (EED)}

To investigate the $E E D$ of the evaluated protocols, we focus on how the average delivery delay varies from the packet was generated at the source UAV until it reaches the destination UAV, including the time of the route discovery if the protocol requires it. As shown in Figures 14(b) and 14(e), the average $E E D$ of $\mathrm{ECaD}$ and MPGR is $\approx 20 \%$ lower than those of all other protocols. In the case of $\mathrm{ECaD}$, it is due to the primary selection of the most connected and energy-rich paths which remain still valid for other data transmissions without re-initializing route discoveries, thus saving more time. As for the outcomes of MPGR, in some cases, it is observed that they are more advantageous than those of $\mathrm{ECaD}$, thus, it can be explained by the shortest distances transited by the data packets to the destination minimizing considerably the average delay by employing an enhanced greedy forwarding technique. Nevertheless, we can clearly distinguish the extra time taken by LEPR, CBLADSR, and UVAR-S, which is caused by the unreliable discovery process requiring its re-initialization at each broken path, the time needed to form each cluster, and the inefficient maintenance technique, respectively, thus wasting more time.

\section{Overhead}

In order to calculate the control overhead, the number of all extra routing packets generated during each data transmission is calculated, and then it is divided on the number of successfully received data packets at destinations. Figures 14(c) and 14(f) show that the control overhead of ECaD is lower than those of UVAR-S and LEPR in $80 \%$ of cases. The reason behind the minimization of the control overhead is due to the use of the most connected and energy-rich paths. With more connectedness and energy efficiency, the need for re-discovery is much reduced, and thus it results in less control overhead. This is not the case of LEPR and UVAR-S, which do not take into account the residual energy of the UAVs making the network more vulnerable to sudden disconnections. Therefore, the route discoveries are frequently made resulting in more control overhead. However, for CBLADSR and MPGR, we can see a small amount of control overhead, which is due to the formation of clusters and the exchange of hello packets, respectively.

\section{E. Path stability and data reliability}

Figs 15(a) and 15(b) show the path stability with respect to varying density and speed, respectively. ECaD demonstrates a high stability (i.e., a reduced number of broken paths) compared to that of all other protocols. This is because ECaD, and unlike the other protocols, takes the mobility, the energy consumption of all UAVs, and the delay of delivery into consideration, which are all used as cost metrics to find the 


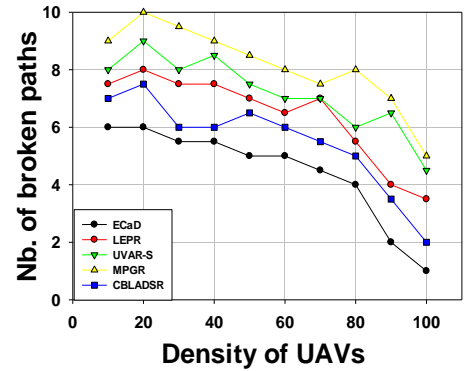

(a)

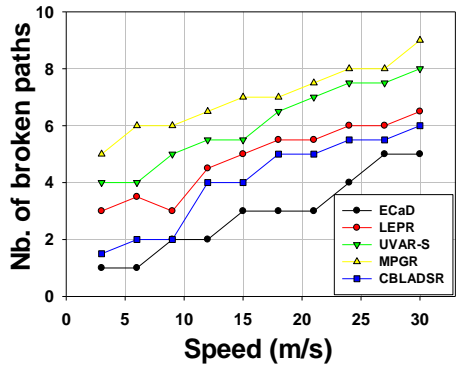

(b)

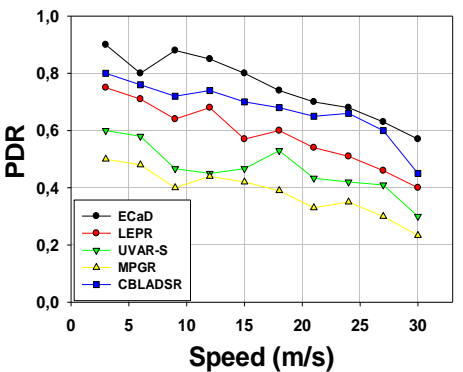

(c)

Fig. 15: Path stability and data reliability when varying the speed and the network scalability. (a) Path stability vs. Density (Speed $=30 \mathrm{~m} / \mathrm{s})$, (b) Path stability vs. Mobility $(\mathrm{UAV}=50)$, and (c) Data reliability vs. Mobility (UAVs=50).

best routing path. In Figure 15(b), it is observed that as the speeds of UAVs are increased, the path stability deteriorates (i.e., higher rate of path failures occurs). Nevertheless, the paths selected by $\mathrm{ECaD}$ have much longer durations than those established by the other protocols, which results in fewer path breaks. According to the results of Figure 15(c), it is shown that the data reliability achieved by $\mathrm{ECaD}$ is better than the other protocols. This is due to the awareness of the movement information and the energy capacity of all UAVs, which increases the delivery ratio. The major enhancement in terms of data reliability appears evident, especially with high mobility situations proving again the strong achievement of $\mathrm{ECaD}$.

\section{CONCLUSION}

Position-based routing is a promising solution to manage the communications between UAVs. Nevertheless, various challenges can be faced in such networks, such as the high mobility of UAVs, the restricted energy, and the frequent disconnections that may occur. The route discovery process is the most traditional mechanism used to find both the destination's position and the appropriate routing path leading to it. Most of the time, this process is not fully exploited to explore the most connected and durable paths, which can result in frequent disconnections and, hence, may introduce an important overhead affecting seriously the data transmission and increasing the delay of delivery. To overcome these problems, we proposed $\mathrm{ECaD}$, which exploits the discovery phase to both predict link failures prior to their occurrence and achieve a balanced energy consumption among UAVs. In the case of path failures, a maintenance process is applied to explore the possibility of finding alternative solutions while considering the lifetime of the links and the residual energy of UAVs. The performance evaluation of $\mathrm{ECaD}$ is carried out based on simulations. Results clearly show the effectiveness of ECaD in terms of PDR, overhead, and end-to-end delay (see Figures 14 and 15). Furthermore, the energy usage is ideally distributed among UAVs during the functioning of $\mathrm{ECaD}$ (see Figure 12). As future perspectives, we plan to adapt our predictive technique for different mobility models. Also, it is believed that $\mathrm{ECaD}$ should be able to provide a long lifetime and stability for different applications requiring frequent communications between UAVs. Moreover, ECaD can be also crucial support for terrestrial networks, such as vehicular networks, where the assistance of communicating UAVs is required. For instance, the works in [39] and [40], proposed two techniques of relay-node selection with the aim to ensure fast dissemination of messages in a highly dense area and to achieve fast delivery of messages and full coverage of the curving road, respectively. However, important hops can be distinguished in these two techniques, which can be significantly improved by using an aerial UAV network adopting ECaD as a networking model.

\section{REFERENCES}

[1] O. S. Oubbati, A. Lakas, F. Zhou, M. Güneş, and M. B. Yagoubi, "A survey on position-based routing protocols for Flying Ad hoc Networks (FANETs)," Vehicular Communications, vol. 10, pp. 29-56, 2017.

[2] M. Mozaffari, W. Saad, M. Bennis, and M. Debbah, "Mobile unmanned aerial vehicles (UAVs) for energy-efficient internet of things communications," IEEE Transactions on Wireless Communications, vol. 16, no. 11, pp. 7574-7589, 2017.

[3] M. Alzenad, A. El-Keyi, F. Lagum, and H. Yanikomeroglu, "3-D placement of an unmanned aerial vehicle base station (UAV-BS) for energy-efficient maximal coverage," IEEE Wireless Communications Letters, vol. 6, no. 4, pp. 434-437, 2017.

[4] M. Mozaffari, W. Saad, M. Bennis, and M. Debbah, "Unmanned aerial vehicle with underlaid device-to-device communications: Performance and tradeoffs," IEEE Transactions on Wireless Communications, vol. 15, no. 6, pp. 3949-3963, 2016.

[5] A. Bujari, C. E. Palazzi, and D. Ronzani, "A Comparison of Stateless Position-based Packet Routing Algorithms for FANETs," IEEE Transactions on Mobile Computing, vol. 16, no. 11, pp. 7574-7589, 2018.

[6] X. Li and J. Huang, "ABPP: An Adaptive Beacon Scheme for Geographic Routing in FANET," Proceedings of the 18th International Conference on Parallel and Distributed Computing, Applications and Technologies (PDCAT), pp. 293-299, 2017.

[7] Y. Zeng, R. Zhang, and T. J. Lim, "Wireless communications with Unmanned Aerial Vehicles: Opportunities and Challenges," IEEE Communications Magazine, vol. 54, no. 5, pp. 36-42, 2016.

[8] A. Osseiran, F. Boccardi, V. Braun, K. Kusume, P. Marsch, M. Maternia, O. Queseth, M. Schellmann, H. Schotten, H. Taoka et al., "Scenarios for $5 \mathrm{G}$ mobile and wireless communications: the vision of the METIS project," IEEE Communications Magazine, vol. 52, no. 5, pp. 26-35, 2014.

[9] C. Yin, S. Ding, and J. Wang, "Mobile marketing recommendation method based on user location feedback," Human-centric Computing and Information Sciences, vol. 9, no. 1, p. 14, 2019.

[10] I. Bekmezci, O. K. Sahingoz, and Ş. Temel, "Flying ad-hoc networks (FANETs): A survey," Ad Hoc Networks, vol. 11, no. 3, pp. 1254-1270, 2013. 
[11] F. Khelifi, A. Bradai, K. Singh, and M. Atri, "Localization and EnergyEfficient Data Routing for Unmanned Aerial Vehicles: Fuzzy-LogicBased Approach," IEEE Communications Magazine, vol. 56, no. 4, pp. 129-133, 2018.

[12] M. Thammawichai, S. P. Baliyarasimhuni, E. C. Kerrigan, and J. B. Sousa, "Optimizing communication and computation for multi-UAV information gathering applications," IEEE Transactions on Aerospace and Electronic Systems, vol. 54, no. 2, pp. 601-615, 2018.

[13] S. He, K. Xie, W. Chen, D. Zhang, and J. Wen, "Energy-aware routing for SWIPT in multi-hop energy-constrained wireless network," IEEE Access, vol. 6, pp. 17996-18 008, 2018.

[14] J. Wang, C. Ju, Y. Gao, A. K. Sangaiah, and G.-j. Kim, "A PSO based energy efficient coverage control algorithm for wireless sensor networks," Comput. Mater. Contin, vol. 56, pp. 433-446, 2018.

[15] J. Wang, Y. Gao, W. Liu, A. K. Sangaiah, and H.-J. Kim, "Energy Efficient Routing Algorithm with Mobile Sink Support for Wireless Sensor Networks," Sensors, vol. 19, no. 7, p. 1494, 2019.

[16] Q. Tang, K. Yang, J. Wang, Y. Luo, K. Li, and F. Yu, "Wireless Sensor Network MCDS Construction Algorithms with Energy Consideration for Extreme Environments Healthcare," IEEE Access, vol. 7, pp. 2169-3536, 2019.

[17] S. He, K. Xie, K. Xie, C. Xu, and J. Wang, "Interference-Aware Multisource Transmission in Multiradio and Multichannel Wireless Network," IEEE Systems Journal, pp. 1-12, 2019.

[18] C. Perkins, E. Belding-Royer, and S. Das, "Ad hoc on-demand distance vector (AODV) routing," Tech. Rep., 2003.

[19] R. Shirani, M. St-Hilaire, T. Kunz, Y. Zhou, J. Li, and L. Lamont, "On the delay of reactive-greedy-reactive routing in unmanned aeronautical ad-hoc networks," Procedia Computer Science, vol. 10, pp. 535-542, 2012.

[20] O. S. Oubbati, A. Lakas, F. Zhou, M. Günes, N. Lagraa, and M. B. Yagoubi, "Intelligent UAV-Assisted Routing Protocol for Urban VANETs," Computer communications, vol. 107, pp. 93-111, 2017.

[21] L. Lin, Q. Sun, J. Li, and F. Yang, "A novel geographic position mobility oriented routing strategy for UAVs," Journal of Computational Information Systems, vol. 8, no. 2, pp. 709-716, 2012.

[22] L. Lin, Q. Sun, S. Wang, and F. Yang, "A geographic mobility prediction routing protocol for ad hoc UAV network," Proceedings of the IEEE Globecom Workshops (GC Wkshps), pp. 1597-1602, 2012.

[23] S. Rosati, K. Krużelecki, and L. Traynard, "Speed-aware routing for UAV ad-hoc networks," Proceedings of the IEEE Globecom Workshops (GC Wkshps), pp. 1367-1373, 2013.

[24] X. Li and J. Yan, "LEPR: Link Stability Estimation-based Preemptive Routing protocol for Flying Ad Hoc Networks," Proceedings of the IEEE Symposium on Computers and Communications (ISCC), pp. 1079-1084, 2017.

[25] N. Shi and X. Luo, "A novel cluster-based location-aided routing protocol for UAV fleet networks," International Journal of Digital Content Technology and its Applications, vol. 6, no. 18, p. 376, 2012.

[26] W. B. Heinzelman, A. P. Chandrakasan, and H. Balakrishnan, "An application-specific protocol architecture for wireless microsensor networks," IEEE Transactions on wireless communications, vol. 1, no. 4 pp. 660-670, 2002.

[27] F. Aadil, A. Raza, M. F. Khan, M. Maqsood, I. Mehmood, and S. Rho, "Energy Aware Cluster-Based Routing in Flying Ad-Hoc Networks," Sensors (Basel, Switzerland), vol. 18, no. 5, 2018.

[28] Q. Tang, M. Xie, K. Yang, Y. Luo, D. Zhou, and Y. Song, “A Decision Function Based Smart Charging and Discharging Strategy for Electric Vehicle in Smart Grid," Mobile Networks and Applications, pp. 1-10, 2018.

[29] J. Wang, Y. Gao, W. Liu, A. K. Sangaiah, and H.-J. Kim, “An intelligent data gathering schema with data fusion supported for mobile sink in wireless sensor networks," International Journal of Distributed Sensor Networks, vol. 15, no. 3, p. 1550147719839581, 2019.

[30] I. Jawhar, N. Mohamed, J. Al-Jaroodi, D. P. Agrawal, and S. Zhang, "Communication and networking of UAV-based systems: Classification and associated architectures," Journal of Network and Computer Applications, vol. 84, pp. 93-108, 2017.

[31] Z. MacHardy, A. Khan, K. Obana, and S. Iwashina, "V2X Access Technologies: Regulation, Research, and Remaining Challenges," IEEE Communications Surveys \& Tutorials, vol. 1, no. 4, pp. 660-670, 2018.

[32] C. Sommer, D. Eckhoff, R. German, and F. Dressler, "A computationally inexpensive empirical model of IEEE $802.11 \mathrm{p}$ radio shadowing in urban environments," Proceedings of the Eighth IEEE International Conference on Wireless On-Demand Network Systems and Services (WONS), pp. 84-90, 2011.
[33] Y. Chen, N. Zhao, Z. Ding, and M.-S. Alouini, "Multiple UAVs as relays: Multi-hop single link versus multiple dual-hop links," IEEE Transactions on Wireless Communications, vol. 17, no. 9, pp. 6348-6359, 2018.

[34] J. Wang, C. Jiang, Z. Han, Y. Ren, R. G. Maunder, and L. Hanzo, "Taking drones to the next level: Cooperative distributed UnmannedAerial-Vehicular Networks for small and mini drones," IEEE Vehicular Technology Magazine, vol. 12, no. 3, pp. 73-82, 2017.

[35] M. Uddin et al., "Link expiration time-aware routing protocol for UWSNs," Journal of Sensors, vol. 2013, pp. 1-9, 2013.

[36] Y. Zeng and R. Zhang, "Energy-efficient UAV communication with trajectory optimization," IEEE Transactions on Wireless Communications, vol. 16, no. 6, pp. 3747-3760, 2017.

[37] S. M. Mousavi, H. R. Rabiee, M. Moshref, and A. Dabirmoghaddam, "Mobisim: A framework for simulation of mobility models in mobile adhoc networks," Proceedings of the Third IEEE International Conference on Wireless and Mobile Computing, Networking and Communications (WiMOB), pp. 82-82, 2007.

[38] L. Gupta, R. Jain, and G. Vaszkun, "Survey of important issues in UAV communication networks," IEEE Communications Surveys \& Tutorials, vol. 18, no. 2, pp. 1123-1152, 2016.

[39] D. Cao, B. Zheng, B. Ji, Z. Lei, and C. Feng, "A robust distancebased relay selection for message dissemination in vehicular network," Wireless Networks, pp. 1-17, 2018.

[40] D. Cao, Y. Liu, X. Ma, J. Wang, B. Ji, C. Feng, and J. Si, "A RelayNode Selection on Curve Road in Vehicular Networks," IEEE Access, vol. 7, pp. 12 714-12 728, 2019.

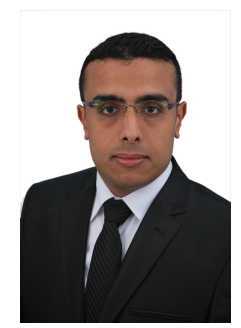

Omar Sami Oubbati is an Associate Professor at the Electronics department, University of Laghouat, Algeria and a Research Assistant in the Computer Science and Mathematics Lab (LIM) at the same university. He received his degree of Engineer (2010), M.Sc. in Computer Engineering (2011), M.Sc. degree (2014), and a PhD in Computer Science (2018). From Oct. 2016 to Oct. 2017, he was a Visiting Student with the Laboratory of Computer Science, University of Avignon, France. His main research interests are in Flying and Vehicular ad hoc networks, Visible light communications, Energy efficiency and Internet of Things (IoT). He is a reviewer in many international journals and a TPC member in many international conferences. He is a member of the IEEE and IEEE Communications Society.

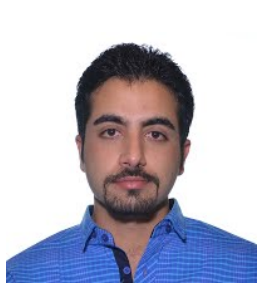

Mohammad Mozaffari received the B.Sc. degree in electrical engineering from the Sharif University of Technology, Iran, and the M.Sc. degree in geomatics engineering from the University of Calgary, Canada. $\mathrm{He}$ is currently pursuing the Ph.D. degree with the Bradley Department of Electrical and Computer Engineering, Virginia Tech. His research interests include wireless communications and statistical signal processing with focus on unmanned aerial vehicle communications, 5G networks, satellite communications, and localization. 


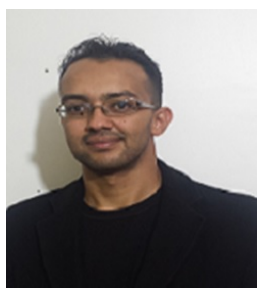

Noureddine Chaib received his $\mathrm{PhD}$ degree in computer science from University of Laghouat and serves as treasurer for IEEE Algeria Section. He is currently an associate professor in the Computer Science Department, the Chief Information Officer and Vice chancellor of University of Laghouat. He is a member of the Computer Science and Mathematics Lab (LIM) and a member of IEEE Vehicular Technology Society. He received the degree of engineering in computer science from University of Laghouat, in 2007, and the degree of Master of engineering in computer science from the University of Batna, in 2011. His research interests include Security, Privacy, Mobile and Vehicular networks and other networking topics.

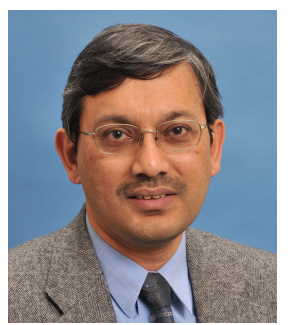

Mohammed Atiquzzaman received the M.S. and $\mathrm{Ph} . \mathrm{D}$. degrees in electrical engineering and electronics from the University of Manchester, U.K., in 1984 and 1987, respectively. He currently holds the Edith J. Kinney Gaylord Presidential Professorship with the School of Computer Science, University of Oklahoma, USA. His research has been funded by the National Science Foundation, National Aeronautics and Space Administration, U.S. Air Force, Cisco, and Honeywell. He co-authored Performance of TCP/IP Over ATM Networks and has authored over 300 refereed publications. His current research interests are in areas of transport protocols, wireless and mobile networks, ad hoc networks, satellite networks, power-aware networking, and optical communications. He CoChaired the IEEE High Performance Switching and Routing Symposium (2003, 2011), IEEE GLOBECOM and ICC (2014, 2012, 2010, 2009, 2007, and 2006), IEEE VTC (2013), and SPIE Quality of Service Over Next Generation Data Networks conferences $(2001,2002$, and 2003). He was the Panels Co-Chair of INFOCOM'05, and has been on the program committee of many conferences, such as INFOCOM, GLOBECOM, ICCCN, ICCIT, Local Computer Networks, and serves on the review panels at the National Science Foundation. He was the Chair of the IEEE Communication Society Technical Committee on Communications Switching and Routing. He received the IEEE Communication Society's Fred W. Ellersick Prize and the NASA Group Achievement Award for outstanding work to further NASA Glenn Research Center's efforts in the area of the Advanced Communications/Air Traffic Management's Fiber Optic Signal Distribution for Aeronautical Communications project. He received from IEEE the 2018 Satellite and Space Communications Technical Recognition Award for valuable contributions to the Satellite and Space Communications scientific community. He also received the 2017 Distinguished Technical Achievement Award from IEEE Communications Society in recognition of outstanding technical contributions and services in the area of communications switching and routing. He is the Editor in-Chief of Journal of Networks and Computer Applications, the founding Editorin-Chief of Vehicular Communications, and serves/served on the editorial boards of many journals, including IEEE Communications Magazine, Real Time Imaging Journal, International Journal of Communication Networks and Distributed Systems, Journal of Sensor Networks, and International Journal of Communication Systems.

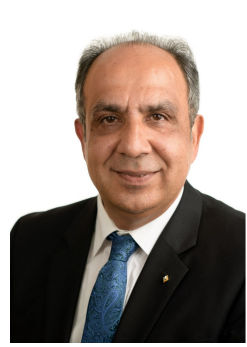

Abbas Jamalipour is the Professor of Ubiquitous Mobile Networking at the University of Sydney, Australia, and holds a PhD in Electrical Engineering from Nagoya University, Japan. He is a Fellow of the Institute of Electrical, Information, and Communication Engineers (IEICE) and the Institution of Engineers Australia, an ACM Professional Member, and an IEEE Distinguished Lecturer. He has authored six technical books, eleven book chapters, over 450 technical papers, and five patents, all in the area of wireless communications. He disseminated the fundamental concepts of the next generation networks and convergence networks as well as the integration of WLAN and cellular networks. He was the Editor-in-Chief IEEE Wireless Communications (2006-08), Vice President-Conferences (2012-13) and a member of Board of Governors of the IEEE Communications Society, and has been an editor for several journals. He has held positions of the Chair of the Communication Switching and Routing and the Satellite and Space Communications Technical Committees and Vice Director of the Asia Pacific Board, in ComSoc. He was a General Chair or Technical Program Chair for a number of conferences, including IEEE ICC, GLOBECOM, WCNC and PIMRC. Dr. Jamalipour is an elected member of the Board of Governors (2014-16 and 2017-19), Executive Vice-President, Chair of Fellow Evaluation Committee, and the Editor-in-Chief of the Mobile World, IEEE Vehicular Technology Society, a voting member of the IEEE Communications Society GITC and IEEE WCNC Steering Committee, and a member of the ComSoc Education Board, and Conference Boards. He is the recipient of a number of prestigious awards such as the 2016 IEEE ComSoc Distinguished Technical Achievement Award in Communications Switching and Routing, the 2010 Royal Academy of Engineering UK Distinguished Fellowship, the 2006 IEEE ComSoc Distinguished Contribution to Satellite Communications Award, 2010 IEEE ComSoc Harold Sobol Award, and the 2006 IEEE ComSoc Best Tutorial Paper Award, and ten best paper awards. $\mathrm{He}$ is one of the most cited researchers in the field of mobile, cellular, and satellite networks with over 10000 citations. 